\title{
Cluster mutation via quiver representations
}

\author{
Aslak Bakke Buan*, Bethany R. Marsh and Idun Reiten
}

Dedicated to Maria Inés Platzeck on the occasion of her 60th birthday

\begin{abstract}
Matrix mutation appears in the definition of cluster algebras of Fomin and Zelevinsky. We give a representation theoretic interpretation of matrix mutation, using tilting theory in cluster categories of hereditary algebras. Using this, we obtain a representation theoretic interpretation of cluster mutation in case of acyclic cluster algebras.
\end{abstract}

Mathematics Subject Classification (2000). Primary 16G20, 16G70; Secondary 13A99, $05 \mathrm{E} 99$.

Keywords. Cluster algebras, cluster categories, tilting theory.

\section{Introduction}

This paper was motivated by the interplay between the recent development of the theory of cluster algebras defined by Fomin and Zelevinsky in [FZ1] (see [Z] for an introduction) and the subsequent theory of cluster categories and cluster-tilted algebras [BMRRT], [BMR]. Our main results can be considered to be interpretations within cluster categories of important concepts in the theory of cluster algebras.

Cluster algebras were introduced in order to explain the connection between the canonical basis of a quantised enveloping algebra as defined by Kashiwara and Lusztig and total positivity for algebraic groups. It was also expected that cluster algebras should model the classical and quantised coordinate rings of varieties associated to algebraic groups - see [BFZ] for an example of this phenomenon (double Bruhat cells). Cluster algebras have been used to define generalisations of the Stasheff polytopes (associahedra) to other Dynkin types [CFZ], [FZ3]; consequently there are likely to be interesting links with operad theory. They have been used to provide the solution [FZ3] of a conjecture of Zamolodchikov concerning $Y$-systems, a class of

\footnotetext{
*Aslak Bakke Buan was supported by a grant from the Norwegian Research Council
} 
functional relations important in the theory of the thermodynamic Bethe Ansatz, as well as solution [FZ4] of various recurrence problems involving Laurent polynomials, including a conjecture of Gale and Robinson on the integrality of generalised Somos sequences. Here the remarkable Laurent properties of the distinguished generators of a cluster algebra play an important role. Cluster algebras have also been related to Poisson geometry [GSV1], Teichmüller spaces [GSV2], positive spaces and stacks [FG], dual braid monoids [BES], ad-nilpotent ideals of a Borel subalgebra of a simple Lie algebra $[\mathrm{P}]$ as well as representation theory, see amongst others [BMRRT], [BMR], [CC], [CCS1], [CCS2], [MRZ].

A cluster algebra (without coefficients) is defined via a choice of free generating set $\underline{x}=\left\{x_{1}, \ldots, x_{n}\right\}$ in the field $\mathcal{F}$ of rational polynomials over $\mathbb{Q}$ and a skewsymmetrizable integer matrix $B$ indexed by the elements of $\underline{x}$. The pair $(\underline{x}, B)$, called a seed, determines the cluster algebra as a subring of $\mathcal{F}$. More specifically, for each $i=1, \ldots, n$, a new seed $\mu_{i}(\underline{x}, B)=\left(\underline{x}^{\prime}, B^{\prime}\right)$ is obtained by replacing $x_{i}$ in $\underline{x}$ by $x_{i}{ }^{\prime} \in \mathcal{F}$, where $x_{i}{ }^{\prime}$ is obtained by a so-called exchange multiplication rule and $B^{\prime}$ is obtained from $B$ by applying so-called matrix mutation at row/column $i$. Mutation in any direction is also defined for the new seed, and by iterating this process one obtains a countable (sometimes finite) number of seeds. For a seed $(\underline{x}, B)$, the set $\underline{x}$ is called a cluster, and the elements in $\underline{x}$ are called cluster variables. The desired subring of $\mathcal{F}$ is by definition generated by the cluster variables.

It is an interesting problem to try to find a categorical/ module theoretical setting with a nice interpretation of the concepts of clusters and cluster variables, and of the matrix mutation and multiplication exchange rule for cluster variables. For the case of acyclic cluster variables so-called cluster categories were introduced as a candidate for such a model [BMRRT]. Skew-symmetric matrices are in one-one correspondence with finite quivers with no loops or cycles of length two, and the corresponding cluster algebra is called acyclic if there is a seed $(\underline{x}, B)$ such that $B$ corresponds to a quiver $Q$ without oriented cycles. There is then, for a field $K$, an associated finite dimensional path algebra $K Q$. The corresponding cluster category $\mathcal{C}$ is defined in [BMRRT] as a certain quotient of the bounded derived category of $K Q$, which is shown to be canonically triangulated by [K]. In [BMRRT] (cluster-)tilting theory is developed in $\mathcal{C}$, with emphasis on connections to cluster algebras. The analogs of clusters are (cluster-)tilting objects, and the analogs of cluster variables are exceptional objects. In case $Q$ is a Dynkin quiver, it was shown in [BMRRT] that there is a one-one correspondence between cluster variables and exceptional objects in $\mathcal{C}$ (in this case all indecomposables are exceptional) which takes clusters to tilting objects. This was conjectured to hold more generally.

In this paper we show that also the matrix mutation for cluster algebras has a nice interpretation within cluster categories, in terms of the associated cluster-tilted algebras, investigated in [BMR]. Cluster-tilted algebras are endomorphism algebras of tilting objects in cluster categories. It follows from our results that the quivers of 
the cluster-tilted algebras arising from a given cluster category are exactly the quivers corresponding to the exchange matrices of the associated cluster algebra. This has further applications to cluster algebras (see [BR]). Another main result of this paper is an interpretation within cluster categories of the exchange multiplication rule of an (acyclic) cluster algebra. So, together with the results from [BMRRT], all the major ingredients involved in the construction of acyclic cluster algebras have now been interpreted in the cluster category.

Tilting theory for hereditary algebras has been a central topic within representation theory since the early eighties. This involves the study of tilted algebras, and various generalisations. An important motivation for this theory was to compare the representation theory of hereditary algebras with the representation theory of other homologically more complex algebras. The main result of [BMR] is also in this spirit, showing a close connection between the representation theory of cluster-tilted algebras and hereditary algebras. It is the hope of the authors that our "dictionary" also can be used to obtain further developments in the representation theory of finite dimensional algebras. Also new links between this field and other fields of mathematics can be expected, having in mind the influence of cluster algebras on other areas.

In [CCS1] an alternative description of the cluster category is given for type $A$. The cluster category was also the motivation for a Hall-algebra type definition of a cluster algebra of finite type [CC], [CK].

The paper is organised as follows. In Section 1 we give some preliminaries, allowing us to state the main result more precisely. Most of the necessary background on cluster algebras is however postponed until later (Section 6), since most of the paper does not involve cluster algebras. In Section 2 we prove the following: If $\Gamma$ is clustertilted, then so is $\Gamma / \Gamma e \Gamma$ for an idempotent $e$ in $\Gamma$. This is an essential ingredient in the proof of the main result, and also an interesting fact in itself. In Section 3 some consequences of this are given. In Section 4 we prepare for the proof of our main result. This involves studying cluster-tilted algebras of rank 3, and a crucial result of Kerner [Ke] on hereditary algebras. The main result is proved in Section 5, while Section 6 deals with the connection to cluster algebras, including necessary background.

The results of this paper have been presented at conferences in Uppsala (June 2004), Mexico (August 2004) and Northeastern University (October 2004).

The first named author spent most of 2004 at the University of Leicester, and would like to thank the Department of Mathematics, and especially Bethany R. Marsh, for their kind hospitality. We would like to thank the referee for pointing out an error in an earlier version of this paper, and Bernhard Keller and Otto Kerner for helpful comments and conversations. 


\section{Preliminaries}

1.1. Finite-dimensional algebras. In this section let $\Lambda$ be a finite dimensional $K$ algebra, where $K$ is a field. Then $1_{\Lambda}=e_{1}+e_{2}+\cdots+e_{n}$, where all $e_{i}$ are primitive idempotents. We always assume that $\Lambda$ is basic, that is, $\Lambda e_{i} \not \Lambda e_{j}$ when $i \neq j$. There are then (up to isomorphism) $n$ indecomposable projective $\Lambda$-modules, given by $\Lambda e_{i}$, and $n$ simple modules, given by $\Lambda e_{i} / \underline{\mathrm{r}} e_{i}$, where $\underline{\mathrm{r}}$ is the Jacobson radical of $\Lambda$.

If $K$ is an algebraically closed field, then there is a finite quiver $Q$, such that $\Lambda$ is isomorphic to $K Q / I$, where $K Q$ is the path-algebra, and $I$ is an admissible ideal, that is there is some $m$, such that $\underline{\mathrm{r}}^{m} \subseteq I \subseteq \underline{\mathrm{r}}^{2}$. We call $Q$ the quiver of $\Lambda$. In case $\Lambda$ is hereditary, the ideal $I$ is 0 . For the rest of this paper, except in Section 2, we always assume that $K$ is algebraically closed.

The category mod $\Lambda$ of finite dimensional left $\Lambda$-modules is an abelian category having almost split sequences. In case $\Lambda$ is hereditary there is a translation functor $\tau$, which is defined on all modules with no projective (non-zero) direct summands.

The bounded derived category of $\Lambda$, denoted $D^{b}(\bmod \Lambda)$, is a triangulated category, with suspension given by the shift-functor [1], which is an autoequivalence. We denote its inverse by [-1]. In this paper, we only consider derived categories of hereditary algebras $H$. They have an especially nice structure, since the indecomposable objects are given by shifts of indecomposable modules. In this case we also have a translation functor $\tau: D^{b}(\bmod H) \rightarrow D^{b}(\bmod H)$, extending the functor mentioned above. We have almost split triangles $A \rightarrow B \rightarrow C \rightarrow$ in $D^{b}(\bmod H)$, where $\tau C=A$, for each indecomposable $C$ in $\mathscr{D}=D^{b}(\bmod H)$. We also have the formula $\operatorname{Hom}_{\mathscr{D}}(X, \tau Y) \simeq D \operatorname{Ext}_{\mathscr{D}}^{1}(Y, X)$, see $[\mathrm{H}]$. Here $D$ denotes the ordinary duality for finite-dimensional algebras. Let $H$ be a hereditary finite-dimensional algebra. Then a module $T$ in mod $H$ is called a tilting module if $\operatorname{Ext}_{H}^{1}(T, T)=0$ and $T$ has, up to isomorphism, $n$ indecomposable direct summands. The endomorphism ring $\operatorname{End}_{H}(T)^{\mathrm{op}}$ is called a tilted algebra.

For an algebra $\Gamma$, it will be convenient to call the quiver of $\Gamma$, what is usually called the quiver of $\Gamma^{\mathrm{op}}$

See $[A R S]$ and $[R]$ for further information on the representation theory of finite dimensional algebras and almost split sequences.

1.2. Approximations. Let $\varepsilon$ be an additive category, and $\mathcal{X}$ a full subcategory. Let $E$ be an object in $\mathcal{E}$. If there is an object $X$ in $\mathcal{X}$, and a map $f: X \rightarrow E$, such that for every object $X^{\prime}$ in $\mathcal{X}$ and every map $g: X^{\prime} \rightarrow E$, there is a map $h: X^{\prime} \rightarrow X$, such that $g=f h$, then $f$ is called a right $\mathcal{X}$-approximation [AS]. The approximation map $f: X \rightarrow E$ is called minimal if no non-zero direct summand of $X$ is mapped to 0 . The concept of (minimal) left $X$-approximations is defined dually. If there is a field $K$, such that $\operatorname{Hom}_{\mathscr{E}}(X, Y)$ is finite dimensional over $K$, for all $X, Y \in \mathcal{E}$, and 
if $\mathcal{X}=$ add $M$ for an object $M$ in $\mathcal{E}$, then (minimal) left and right $\mathcal{X}$-approximations always exist. Here, for an object $M$ in any additive category, we let add $M$ denote the smallest full additive subcategory closed under direct sums and containing $M$.

1.3. Cluster categories and cluster-tilted algebras. We remind the reader of the basic definitions and results from [BMRRT]. Let $H$ be a hereditary algebra, and let $\mathscr{D}=D^{b}(\bmod H)$ be the bounded derived category.

The cluster category is defined as the orbit category $\mathcal{C}_{H}=\mathscr{D} / F$, where $F=$ $\tau^{-1}[1]$. The objects of $\mathcal{C}_{H}$ are the same as the objects of $\mathcal{D}$, but maps are given by

$$
\operatorname{Hom}_{\mathcal{C}}(X, Y)=\bigsqcup_{i} \operatorname{Hom}_{\mathscr{D}}\left(X, F^{i} Y\right) .
$$

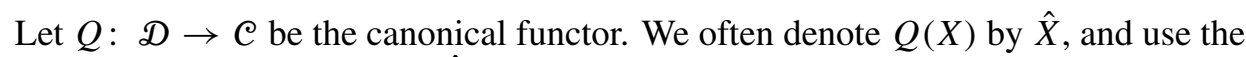
same notation for maps. Let $\hat{X}$ be an indecomposable object in the cluster-category. We call $\bmod H \vee$ add $H[1]=\bmod H \vee H[1]$ the standard domain.

There is (up to isomorphism) a unique object $X$ in $\bmod H \vee H[1] \subseteq \mathscr{D}$ such that $Q(X)=\hat{X}$.

Assume $X_{1}, X_{2}$ are indecomposable in the standard domain, then a map $\hat{f}: \widehat{X_{1}} \rightarrow$ $\widehat{X_{2}}$, can uniquely be written as a sum of maps $\hat{f}_{1}+\hat{f}_{2}+\cdots+\hat{f}_{r}$, such that $f_{i}$ is in $\operatorname{Hom}_{\mathcal{D}}\left(X_{1}, F^{d_{i}} X_{2}\right)$, for integers $d_{i}$. In this case $d_{i}$ is called the degree of $\hat{f}_{i}$.

The following summarises properties about cluster categories that will be freely used later.

Theorem 1.1. Let $H$ be a hereditary algebra, and $\mathcal{C}_{H}$ the cluster category of $H$. Then

(a) $\mathcal{C}_{H}$ is a Krull-Schmidt category and $Q$ preserves indecomposable objects;

(b) $\mathcal{C}_{H}$ is triangulated and $Q$ is exact;

(c) $\mathcal{C}_{H}$ has AR-triangles and $Q$ preserves AR-triangles.

Proof. (b) is due to Keller [K], while (a) and (c) are proved in [BMRRT].

Let us now fix a hereditary algebra $H$, and assume it has, up to isomorphism, $n$ simple modules. A cluster tilting object (or for short, tilting object) in the cluster category is an object $T$ with $\operatorname{Ext}_{\mathcal{C}}^{1}(T, T)=0$, and with $n$ non-isomorphic indecomposable direct summands. Two tilting objects $T$ and $T^{\prime}$ are said to be equivalent if and only if add $T=\operatorname{add} T^{\prime}$. We only consider tilting objects up to equivalence, and therefore we always assume that if $T=\bigsqcup_{i} T_{i}$ is a tilting object, with each $T_{i}$ indecomposable, then $T_{i} \not$ T $T_{j}$ for $i \neq j$.

There is a natural embedding of the module category into the bounded derived category, which extends to an embedding of the module category into $\mathcal{C}$. This embedding is in general not full. It was shown in [BMRRT] that the image of a tilting 
module in mod $H$ is a tilting object in $\mathcal{C}_{H}$. It was also shown that if we choose a tilting object $T$ in $\mathcal{C}_{H}$, then there is a hereditary algebra $H^{\prime}$ and an equivalence $D^{b}\left(H^{\prime}\right) \rightarrow D^{b}(H)$, such that $T$ is the image of a tilting module, under the embedding of $\bmod H^{\prime}$ into $\mathcal{C}_{H^{\prime}} \simeq \mathcal{C}_{H}$.

If $\bar{T} U X$ is a tilting object, and $X$ is indecomposable, then $\bar{T}$ is called an almost complete tilting object.

The following was shown in [BMRRT].

Theorem 1.2. Let $\bar{T}$ be an almost complete tilting object in $\mathcal{C}_{H}$. Then there are exactly two complements $M$ and $M^{*}$. There are uniquely defined non-split triangles

$$
M^{*} \rightarrow B \rightarrow M \rightarrow
$$

and

$$
M \rightarrow B^{\prime} \rightarrow M^{*} \rightarrow
$$

The maps $B \rightarrow M$ and $B^{\prime} \rightarrow M^{*}$ are minimal right add $\bar{T}$-approximations, and the maps $M^{*} \rightarrow B$ and $M \rightarrow B^{\prime}$ are minimal left add $\bar{T}$-approximations.

The endomorphism ring $\operatorname{End}_{\mathcal{C}}(T)^{\text {op }}$ of a tilting object in $\mathcal{C}$ is called a cluster-tilted algebra. Using the notation of Theorem 1.2, we want to compare the quivers of the endomorphism rings $\Gamma=\operatorname{End}_{\mathcal{C}}(\bar{T} \amalg M)^{\mathrm{op}}$ and $\Gamma^{\prime}=\operatorname{End}_{\mathcal{C}}\left(\bar{T} \amalg M^{*}\right)^{\mathrm{op}}$.

1.4. Matrix mutation. Let $X=\left(x_{i j}\right)$ be an $n \times n$-skew-symmetric matrix with integer entries. Choose $k \in\{1,2, \ldots, n\}$ and define a new matrix $\mu_{k}(X)=X^{\prime}=$ $\left(x_{i j}^{\prime}\right)$ by

$$
x_{i j}^{\prime}= \begin{cases}-x_{i j} & \text { if } k=i \text { or } k=j, \\ x_{i j}+\frac{\left|x_{i k}\right| x_{k j}+x_{i k}\left|x_{k j}\right|}{2} & \text { otherwise. }\end{cases}
$$

The matrix $\mu_{k}(X)=X^{\prime}$ is called the mutation of $X$ in direction $k$, and one can show that

- $\mu_{k}(X)$ is skew-symmetric, and

- $\mu_{k}\left(\mu_{k}(X)\right)=X$.

Matrix mutation appears in the definition of cluster algebras by Fomin and Zelevinsky [FZ1].

1.5. Main result. At this point, we have the necessary notation to state the main result of this paper. There are no loops in the quiver of a cluster-tilted algebra [BMRRT], and we also later show that there are no (oriented) cycles of length two. It follows that one can assign to $\Gamma$ a skew-symmetric integer matrix $X_{\Gamma}$. Actually, there is a 1-1 correspondence between the skew-symmetric integer matrices and quivers 
with no loops and no cycles of length two. Fixing an ordering of the vertices of the quiver, this 1-1 correspondence determines mutations $\mu_{k}$ also on finite quivers (with no loops and no cycles of length 2). The following will be proved in Section 5 . The notation is as earlier in this section, especially the field $K$ is algebraically closed.

Theorem 1.3. Let $\bar{T}$ be an almost complete tilting object with complements $M$ and $M^{*}$ and let $\Gamma=\operatorname{End}_{\mathcal{C}}(\bar{T} \amalg M)^{\mathrm{op}}$ and $\Gamma^{\prime}=\operatorname{End}_{\mathcal{C}}\left(\bar{T} \amalg M^{*}\right)^{\mathrm{op}}$. Let $k$ be the vertex of $\Gamma$ corresponding to $M$. Then the quivers $Q_{\Gamma}$ and $Q_{\Gamma^{\prime}}$, or equivalently the matrices $X_{\Gamma}=\left(x_{i j}\right)$ and $X_{\Gamma^{\prime}}=\left(x_{i j}^{\prime}\right)$, are related by the formulas

$$
x_{i j}^{\prime}= \begin{cases}-x_{i j} & \text { if } k=i \text { or } k=j, \\ x_{i j}+\frac{\left|x_{i k}\right| x_{k j}+x_{i k}\left|x_{k j}\right|}{2} & \text { otherwise. }\end{cases}
$$

This is the central result from which the connections with cluster algebras mentioned in the introduction follow. An independent proof of Theorem 1.3 in the case of finite representation type is given by Caldero, Chapoton and Schiffler [CCS2].

\section{Factors of cluster-tilted algebras}

In this section, our main result is that for any cluster-tilted algebra $\Gamma$, and any primitive idempotent $e$, the factor-algebra $\Gamma / \Gamma e \Gamma$ is in a natural way also a cluster-tilted algebra. This will give us a powerful reduction-technique, which is of independent interest, and which we use in the proof of our main result in this paper.

Suppose that $\Gamma$ is the endomorphism algebra of a tilting object $T$ in the cluster category corresponding to a hereditary algebra $H$. The main idea of the proof is to show that if we localise $\mathscr{D}=D^{b}(\bmod H)$ at the smallest thick subcategory containing a fixed indecomposable summand $M$ of $T$, then we obtain a category triangle-equivalent to the derived category of a hereditary algebra $H^{\prime}$. The factoralgebra $\Gamma / \Gamma e \Gamma$ (where $e$ is the primitive idempotent of $\Gamma$ corresponding to $M$ ) is then shown to be isomorphic to the endomorphism algebra of a tilting object in the cluster category corresponding to $H^{\prime}$.

2.1. Localisation of triangulated categories. We review the basics of localisation in triangulated categories. Let $\mathcal{T}$ be a triangulated category. A subcategory $\mathcal{M}$ of $\mathcal{T}$ is called a thick subcategory of $\mathcal{T}$ if it is a full triangulated subcategory of $\mathcal{T}$ closed under taking direct summands.

When $\mathcal{M}$ is a thick subcategory of $\mathcal{T}$, one can form a new triangulated category $\mathcal{T}_{\mathcal{M}}=\mathcal{T} / \mathcal{M}$, and there is a canonical exact functor $L_{\mathcal{M}}: \mathcal{T} \rightarrow \mathcal{T}_{\mathcal{M}}$. See [Ric] and [V] for details. 
For every $M^{\prime}$ in $\mathcal{M}$, we have $L_{\mathcal{M}}\left(M^{\prime}\right)=0$, and $L_{\mathcal{M}}$ is universal with respect to this property. We also have the following.

Lemma 2.1. Assume $\mathcal{T}$ is a triangulated category, and $\mathcal{M}$ is a thick subcategory of $\mathcal{T}$. Then, for any map $f$ in $\mathcal{T}$ we have $L_{\mathcal{M}}(f)=0$ if and only if $f$ factors through an object in $\mathcal{M}$.

We will need the following result of Verdier [V, Ch. 2, 5-3], [V2]:

Proposition 2.2. Let $\mathcal{T}$ be a triangulated category with thick subcategory $\mathcal{M}$, and let $\mathcal{T}_{\mathcal{M}}$ be the quotient category with quotient functor $L_{\mathcal{M}}: \mathcal{T} \rightarrow \mathcal{T}_{\mathcal{M}}$. Fix an object $Y$ of $\mathcal{T}$. Then every morphism from an object of $\mathcal{M}$ to $Y$ is zero if and only if for every object $X$ of $\mathcal{T}$ the canonical map

$$
\operatorname{Hom}_{\mathcal{T}}(X, Y) \rightarrow \operatorname{Hom}_{\tau_{\mathcal{M}}}\left(L_{\mathcal{M}}(X), L_{\mathcal{M}}(Y)\right)
$$

is an isomorphism.

In particular, we note that this implies that $L_{\mathcal{M}}$ is fully faithful on the full subcategory of $\mathcal{T}$ with objects given by those objects of $\mathcal{T}$ which have only zero morphisms from objects of $\mathcal{M}$.

2.2. Equivalences of module categories. Let $H$ be a hereditary algebra and $M$ an indecomposable $H$-module with $\operatorname{Ext}_{H}^{1}(M, M)=0$. Then there is (up to isomorphism) a unique module $E$ with the following properties:

B1) $E$ is a complement of $M$ (that is, $E \amalg M$ is a tilting module).

B2) For any module $X$ in $\bmod H$, we have that $\operatorname{Ext}_{H}^{1}(M, X)=0$ implies also $\operatorname{Ext}_{H}^{1}(E, X)=0$.

This is due to Bongartz [B], and the module $E$ is sometimes called the Bongartzcomplement of $M$. For a module $X$ in mod $H$, we denote by $X^{\perp}$ the full subcategory of mod $H$ with objects $Y$ satisfying $\operatorname{Ext}_{H}^{1}(X, Y)=0$. If $T$ is a tilting module, then it is well known that $T^{\perp}=\operatorname{Fac} T$, where Fac $T$ is the full subcategory of all modules that are factors of objects in add $T$. Note that B2) can be reformulated as $M^{\perp}=(M \amalg E)^{\perp}$.

The following result can be found in $[\mathrm{H}]$ and [HRS].

Proposition 2.3. (a) Assume $M$ is an indecomposable non-projective $H$-module with $\operatorname{Ext}_{H}^{1}(M, M)=0$, and let $E$ be the complement as above. Then the endomorphism ring $H^{\prime}=\operatorname{End}_{H}(E)^{\mathrm{op}}$ is hereditary, and $\operatorname{Hom}_{H}(M, E)=0$.

(b) Let $U$ denote the full subcategory of $\bmod H$ with objects $X$ satisfying

$$
\operatorname{Hom}_{H}(M, X)=0=\operatorname{Ext}_{H}^{1}(M, X) .
$$


Then $U$ is an exact subcategory of $\bmod H$ and the functor $\operatorname{Hom}_{H}(E,-)$ from $\bmod H$ to $\bmod H^{\prime}$ restricts to an exact equivalence between $U$ and $\bmod H^{\prime}$.

We note that the above result does not hold in general in the case when $M$ is projective. For example, consider the quiver of type $A_{3}$ with vertices 1,2 and 3 and arrows from 1 to 2 and 2 to 3 . Let $M=P_{2}$. Then $E=P_{1} \oplus P_{3}$ and $\operatorname{End}_{H}(E)^{o p}$ has three indecomposable objects while $U$ has only two. The only other complement of $M$ is $E^{\prime}=P_{1} \oplus\left(P_{2} / P_{3}\right)$. Then $\operatorname{Hom}_{H}\left(E^{\prime}, P_{3}\right)=0$ although $P_{3}$ lies in $U$ and is non-zero. So also in this case the functor $\operatorname{Hom}_{H}\left(E^{\prime},\right)$ from $\mathcal{U}$ to $\bmod \operatorname{End}_{H}\left(E^{\prime}\right)^{\text {op }}$ is not an equivalence. However, we will need the following result which is along similar lines for the case when $M$ is projective.

Lemma 2.4. Let $M$ be an indecomposable projective $H$-module with corresponding idempotent $e_{M} \in H$. Let $H^{\prime}=H / H e_{M} H$.

(a) We have $\operatorname{Tor}_{1}^{H}\left(H^{\prime}, U\right)=0$, for any object $U$ in $U$, where $U$ is as defined above.

(b) We have that $U$ is an exact subcategory of $\bmod H$ and the functor $H^{\prime} \otimes_{H}-$ from $\bmod H$ to $\bmod H^{\prime}$ restricts to an exact equivalence between $\mathcal{U}$ and $\bmod H^{\prime}$.

Proof. We have that $\mathcal{U}$ is an exact subcategory of $\bmod H$ as in Proposition 2.3. It is easy to see that the functor $H^{\prime} \otimes_{H}-$ is an equivalence between $U$ and mod $H^{\prime}$. To see that it is exact, we consider the following projective resolution of $H^{\prime}$ as a right $H$-module:

$$
0 \rightarrow H e_{M} H \rightarrow H \rightarrow H^{\prime} \rightarrow 0 .
$$

Applying $-\otimes_{H} U$ to this sequence, where $U$ is an object in $U$, we obtain the long exact sequence:

$$
\operatorname{Tor}_{1}^{H}(H, U) \rightarrow \operatorname{Tor}_{1}^{H}\left(H^{\prime}, U\right) \rightarrow H e_{M} H \otimes_{H} U \rightarrow H \otimes_{H} U \rightarrow H^{\prime} \otimes_{H} U \rightarrow 0 .
$$

Since $H$ is projective, $\operatorname{Tor}_{1}^{H}(H, U)=0$. We also have

$$
H e_{M} H \otimes_{H} U=H \otimes_{H} H e_{M} U=0
$$

since $e_{M} U=0$. It follows that $\operatorname{Tor}_{1}^{H}\left(H^{\prime}, U\right)=0$ and hence that $H^{\prime} \otimes_{H}-$ is an exact functor on $\mathcal{U}$.

2.3. Localising with respect to an exceptional module. Fix a hereditary algebra $H$, and an indecomposable module $M$ in $\bmod H$, with $\operatorname{Ext}_{H}^{1}(M, M)=0$.

Lemma 2.5. Let $\mathcal{M}=\operatorname{add}\{M[i]\}_{i \in \mathbb{Z}}$. Then $\mathcal{M}$ is a thick subcategory in $D^{b}(\bmod H)$.

Proof. Straightforward from the fact that any map between indecomposable objects in $\mathcal{M}$ is either zero or an isomorphism. 
Let $\mathscr{D}=D^{b}(\bmod H)$, let $\mathscr{D}_{\mathcal{M}}$ be the category obtained from $\mathscr{D}$ by localising with respect to $\mathcal{M}$, and let $L_{\mathcal{M}}: \mathscr{D} \rightarrow \mathscr{D}_{\mathcal{M}}$ be the localisation functor. Note that $\mathcal{U}$ is the full subcategory of $\bmod H$ consisting of modules $X$ with $\operatorname{Hom}_{\mathscr{D}}(M, X[i])=0$ for all $i$.

Theorem 2.6. Let $H$ be a hereditary algebra with $n$ simple modules up to isomorphism. Let $M$ be an indecomposable $H$-module with $\operatorname{Ext}_{H}^{1}(M, M)=0$, and let $\mathcal{M}$ denote the thick subcategory generated by $M$. Then $\mathscr{D}_{\mathcal{M}}$ is equivalent to the derived category of a hereditary algebra with $n-1$ simple modules (up to isomorphism).

To prove this we show that $\mathscr{D}_{\mathcal{M}}$ is equivalent to the subcategory $\mathscr{D}_{0}=\operatorname{add}\{X[i] \in$ $\mathscr{D} \mid X \in \mathcal{U}, i \in \mathbb{Z}\}$ of $\mathscr{D}$, and that $\mathscr{D}_{0}$ is equivalent to the derived category of a hereditary algebra with $n-1$ simple modules. This is the content of the following three propositions. We usually denote the object $L_{\mathcal{M}}(X)$ by $\tilde{X}$.

Proposition 2.7. In the setting of Theorem 2.6, the localisation functor $L_{\mathcal{M}}$ induces an equivalence $\mathscr{D}_{0} \rightarrow \mathbb{D}_{\mathcal{M}}$.

Proof. First note that by Proposition 2.2 we have that $L_{\mathcal{M}}: \mathscr{D}_{0} \rightarrow \mathscr{D}_{\mathcal{M}}$ is fully faithful. Any object in $\mathscr{D}_{\mathcal{M}}$ is of the form $L_{\mathcal{M}}(X)$ for some object $X$ in $\mathscr{D}$. Let $\widetilde{X}$ be an arbitrary object in $\mathscr{D}_{\mathcal{M}}$ (where $X$ is in $\left.\mathscr{D}\right)$. Then consider the minimal right $\mathcal{M}$-approximation $M_{X} \rightarrow X$, and the induced triangle $M_{X} \rightarrow X \rightarrow X_{0} \rightarrow$. It is clear that $\widetilde{X}=\widetilde{X_{0}}$. We claim that $X_{0}$ is in $\mathscr{D}_{0}$, that is $\operatorname{Hom}_{\mathscr{D}}\left(M, X_{0}[i]\right)=0$ for all $i$. To see this, consider the long exact sequence obtained by applying $\operatorname{Hom}_{\mathscr{D}}(M$, ) to the triangle $M_{X} \rightarrow X \rightarrow X_{0} \rightarrow$. For any $i$, the map $\operatorname{Hom}_{\mathcal{D}}\left(M, M_{X}[i]\right) \rightarrow$ $\operatorname{Hom}_{\mathscr{D}}(M, X[i])$ is an epimorphism, since $M_{X} \rightarrow X$ is a right $\mathcal{M}$-approximation. The map is injective since any element in $\operatorname{Hom}_{\mathscr{D}}\left(M, M_{X}[i]\right)$ is either zero or an isomorphism. Thus, $X_{0}$ is in $\mathscr{D}_{0}$. This completes the proof that $L_{\mathcal{M}}$ induces an equivalence $\mathscr{D}_{0} \rightarrow \mathscr{D}_{\mathcal{M}}$.

The next result is an extension of Proposition 2.3 to the setting of derived categories.

Proposition 2.8. In the setting of Theorem 2.6, assume $M$ is non-projective. Let $E$ be the Bongartz-complement of $M$, and let $H^{\prime}=\operatorname{End}_{H}(E)^{o p}$. Then $\mathbb{R H o m}(E$, ) induces an equivalence $\mathscr{D}_{0} \rightarrow \mathscr{D}^{\prime}=D^{b}\left(\bmod H^{\prime}\right)$.

Proof. Recall that $U \subset M^{\perp}=(M \amalg E)^{\perp}$. This implies that for $X \in U$, we have that $\mathbb{R} \operatorname{Hom}(E, X)$ is concentrated in degree zero with zero-term $\operatorname{Hom}_{H}(E, X)$. Since $\operatorname{Hom}_{H}(E$,$) is a dense functor from U$ to $\bmod H^{\prime}$, and $\mathbb{R} \operatorname{Hom}(E$,$) commutes with$ [1], it follows that $\mathbb{R} \operatorname{Hom}\left(E\right.$, ) restricted to $\mathscr{D}_{0}$ is dense. 
Assume $X, Y$ are indecomposable objects in the same degree in $\mathscr{D}_{0}$. By the above it now follows directly from Proposition 2.3 that

$$
\operatorname{Hom}_{\mathscr{D}}(X, Y) \simeq \operatorname{Hom}_{D^{\prime}}(\mathbb{R} \operatorname{Hom}(E, X), \mathbb{R} \operatorname{Hom}(E, Y)) .
$$

We also need to show that

$$
\operatorname{Hom}_{\mathscr{D}}(X, Y[1]) \simeq \operatorname{Hom}_{\mathcal{D}^{\prime}}(\mathbb{R} \operatorname{Hom}(E, X), \mathbb{R} \operatorname{Hom}(E, Y[1])) .
$$

For this note that by Proposition 2.3, the equivalence $\operatorname{Hom}_{H}(E):, U \rightarrow \bmod H^{\prime}$ is exact, and that the embedding $U \hookrightarrow \bmod H$ is exact. This implies that

$$
\begin{aligned}
\operatorname{Hom}_{\mathscr{D}}(X, Y[1]) & \simeq \operatorname{Hom}_{u}(X, Y[1]) \\
& \simeq \operatorname{Hom}_{D^{\prime}}\left(\operatorname{Hom}_{H}(E, X), \operatorname{Hom}_{H}(E, Y)[1]\right) \\
& \simeq \operatorname{Hom}_{D^{\prime}}(\mathbb{R} \operatorname{Hom}(E, X), \mathbb{R} \operatorname{Hom}(E, Y)[1]) \\
& \simeq \operatorname{Hom}_{D^{\prime}}(\mathbb{R} \operatorname{Hom}(E, X), \mathbb{R} \operatorname{Hom}(E, Y[1])) .
\end{aligned}
$$

Thus the restriction of $\mathbb{R} \operatorname{Hom}(E$,$) to \mathscr{D}_{0}$ is fully faithful. This completes the proof.

Proposition 2.9. In the setting of Theorem 2.6, assume $M$ is projective. Assume $M \simeq H e_{M}$ for the primitive idempotent $e_{M}$ in $H$ and let $H^{\prime}=H / H e_{M} H$. Then $\mathbb{L}\left(H^{\prime} \otimes_{H}-\right)$ induces an equivalence $\mathscr{D}_{0} \rightarrow \mathscr{D}^{\prime}=D^{b}\left(\bmod H^{\prime}\right)$.

Proof. First recall from Lemma 2.4 that $\operatorname{Tor}_{1}^{H}\left(H^{\prime}, U\right)=0$ for any $U$ in $U$. This means that the image $\mathbb{L}\left(H^{\prime} \otimes_{H} U\right)$ is just $H^{\prime} \otimes_{H} U$ concentrated in degree 0 .

It now follows that $\mathbb{L}\left(H^{\prime} \otimes_{H}-\right)$ restricted to $\mathscr{D}_{0}$ is dense, by using that the functor $H^{\prime} \otimes_{H}-: \quad U \rightarrow \bmod H^{\prime}$ is dense and that $\mathbb{L}\left(H^{\prime} \otimes_{H}-\right)$ commutes with [1].

Assume $X, Y$ are indecomposable objects in the same degree in $\mathscr{D}_{0}$. It follows from Lemma 2.4 that

$$
\operatorname{Hom}_{\mathscr{D}}(X, Y) \simeq \operatorname{Hom}_{D^{\prime}}\left(\mathbb{L}\left(H^{\prime} \otimes_{H} X\right), \mathbb{L}\left(H^{\prime} \otimes_{H} Y\right)\right) .
$$

We need also to show that

$$
\operatorname{Hom}_{\mathscr{D}}(X, Y[1]) \simeq \operatorname{Hom}_{D^{\prime}}\left(\mathbb{L}\left(H^{\prime} \otimes_{H} X\right), \mathbb{L}\left(H^{\prime} \otimes_{H} Y[1]\right)\right) .
$$

For this recall that the embedding of $U$ into $\bmod H$ is exact, and that $H^{\prime} \otimes_{H}-$ is exact on $\mathcal{U}$ by Lemma 2.4. Thus it follows that:

$$
\begin{aligned}
\operatorname{Hom}_{\mathscr{D}}(X, Y[1]) & \simeq \operatorname{Hom}_{u}(X, Y[1]) \\
& \simeq \operatorname{Hom}_{D^{\prime}}\left(H^{\prime} \otimes_{H} X, H^{\prime} \otimes_{H} Y[1]\right) \\
& \simeq \operatorname{Hom}_{D^{\prime}}\left(\mathbb{L}\left(H^{\prime} \otimes_{H} X\right), \mathbb{L}\left(H^{\prime} \otimes_{H} Y\right)[1]\right) \\
& \simeq \operatorname{Hom}_{D^{\prime}}\left(\mathbb{L}\left(H^{\prime} \otimes_{H} X\right), \mathbb{L}\left(H^{\prime} \otimes_{H} Y[1]\right)\right) .
\end{aligned}
$$

This shows that the functor is fully faithful and finishes the proof. 
For the remainder of this section, we view the induced equivalence between $\mathscr{D}_{\mathscr{M}}$ and $\mathscr{D}^{\prime}$ as an identification.

2.4. The factor construction. As before, let $M$ be an indecomposable $H$-module with $\operatorname{Ext}_{H}^{1}(M, M)=0$, where $H$ is hereditary, and let $E$ be the Bongartz complement of $M$. We investigate the image of an arbitrary complement $\bar{T}$ of $M$ under the functor $L_{\mathcal{M}}$. For an object $X$ in $\mathscr{D}$, we use the notation $\widetilde{X}=L_{\mathcal{M}}(X)$, as before. Note that $L_{\mathcal{M}}(\bar{T})=L_{\mathcal{M}}(T)=\widetilde{T}$, where $T=\bar{T} \amalg M$.

Lemma 2.10. Let the notation be as above.

(a) $L_{\mathcal{M}}(\bar{T})=\widetilde{T}$ is in $\bmod H^{\prime} \vee H^{\prime}[1]$.

(b) $\operatorname{Hom}_{D^{\prime}}(\widetilde{T}, \widetilde{T}[1])=0$.

Proof. (a) Let $f: M^{\prime} \rightarrow \bar{T}$ be a minimal right $\mathcal{M}$-approximation, and consider the induced triangle:

$$
M^{\prime} \stackrel{f}{\rightarrow} \bar{T} \stackrel{g}{\rightarrow} U_{T} \rightarrow
$$

in $\mathscr{D}$. Since $\operatorname{Hom}_{\mathscr{D}}(M[-1], \bar{T})=0$, we have that $M^{\prime}$ is in add $M$. It is clear that $\widetilde{T} \simeq \widetilde{U_{T}}$. Now, as in the proof of Proposition 2.7, we get that $U_{T}$ is in $\mathcal{D}_{0}$. Here it is clear that $U_{T}=U_{1} \amalg U_{2}$ [1], where $U_{1}=$ Coker $f$ and $U_{2}=\operatorname{Ker} f$ are in $\mathcal{u}$. It is clear that $\widetilde{U}_{1}$ and $\widetilde{U}_{2}$ are $H^{\prime}$-modules. We only need to show that $\widetilde{U}_{2}$ is projective. For an arbitrary $U$ in $U$, we have that $\operatorname{Ext}_{H}^{1}\left(U_{2}, U\right)=0$, since $\operatorname{Ext}_{H}^{1}(M, U)=0$ and $U_{2}$ is a submodule of $M^{\prime}$. Using that $U$ is an exact subcategory of $\bmod H$, and that the equivalence $\underset{\widetilde{T}}{U} \rightarrow \bmod H^{\prime}$ is also exact, it follows that $\widetilde{U_{2}}$ is projective in $\bmod H^{\prime}$. Hence $\widetilde{T} \simeq \widetilde{U_{T}}$ is in $\bmod H^{\prime} \vee H^{\prime}[1]$.

(b) Using again the triangle (1) we obtain the long exact sequence

$$
\operatorname{Hom}_{\mathscr{D}}(\bar{T}, \bar{T}[1]) \rightarrow \operatorname{Hom}_{\mathscr{D}}\left(\bar{T}, U_{T}[1]\right) \rightarrow \operatorname{Hom}_{\mathscr{D}}\left(\bar{T}, M^{\prime}[2]\right) .
$$

Therefore, $\operatorname{Hom}_{\mathscr{D}}\left(\bar{T}, U_{T}[1]\right)=0$. Now, by Proposition 2.2, it follows that $\operatorname{Hom}_{D^{\prime}}\left(\widetilde{T}, \widetilde{U_{T}[1]}\right)=0$ since $U_{T}[1]$ is in $\mathscr{D}_{0}$, and hence $\operatorname{Hom}_{D^{\prime}}(\widetilde{T}, \widetilde{T}[1])=0$.

Denote as before by $F$ the functor $\tau^{-1}[1]: \mathscr{D} \rightarrow \mathscr{D}$. When it is not clear which derived category $\mathscr{D}$ we are dealing with, we will denote this functor by $F_{\mathscr{D}}$ and the functor $\tau^{-1}$ by $\tau_{\mathscr{D}}^{-1}$.

Lemma 2.11. Let $H$ be a hereditary algebra, and let $X$ be an object in $D$ such that $X$ is in $\bmod H \vee H[1]$. Then $\operatorname{Hom}_{\mathscr{D}}(X, X[1])=0$ if and only if $\operatorname{Ext}_{\mathfrak{C}_{H}}^{1}(X, X)=0$. 
Proof. Assume $X$ is in mod $H \vee H[1]$, and let $\hat{X}$ be the image of $X$ in the cluster

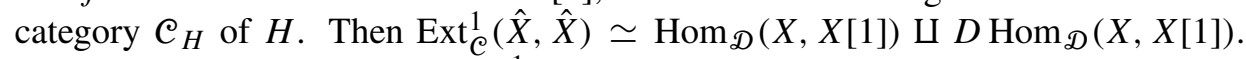
This follows from $\operatorname{Hom}_{\mathscr{D}}\left(X, F^{-1} X[1]\right)=\operatorname{Hom}_{\mathscr{D}}(X, \tau X) \simeq D \operatorname{Hom}_{\mathscr{D}}(X, X[1])$ and the easily checked fact that $\operatorname{Hom}_{\mathscr{D}}\left(X, F^{i} X[1]\right)=0$, whenever $i \notin\{-1,0\}$.

Combining these lemmas, and using that a tilting $H$-module induces a tilting object in the cluster category [BMRRT, 3.3], we obtain the following.

Proposition 2.12. Let $T=M \amalg \bar{T}$ be a tilting $H$-module as before. Then the image $\widehat{T}$ of $\widetilde{T}$ in the cluster category $\mathcal{C}_{H^{\prime}}$ is a tilting object.

Proof. By Lemmas 2.10 and 2.11 we have that $\operatorname{Ext}_{\mathcal{C}_{H^{\prime}}}^{1}(\widehat{T}, \widehat{T})=0$, and that $\widetilde{T}$ is in $\bmod H^{\prime} \vee H^{\prime}[1]$. It follows from Lemma 2.1 that $\widetilde{T}_{a}$ is non-zero when $T_{a}$ is a direct summand in $\bar{T}$. Thus, it suffices to show the following: For two indecomposable nonisomorphic direct summands $T_{a}$ and $T_{b}$ of $\bar{T}$, we have $\widetilde{T}_{a} \not \widetilde{T}_{b}$. We first show that for any map $\alpha: \widetilde{T}_{a} \rightarrow \widetilde{T}_{b}$, there is a map $\beta: T_{a} \rightarrow T_{b}$ such that $L_{\mathcal{M}}(\beta)=\widetilde{\beta}=\alpha$. Maps in $\mathscr{D}^{\prime}$ from $T_{a}$ to $T_{b}$ can be viewed as certain equivalence classes of pairs $\left(\alpha_{1}, \alpha_{2}\right)$ of maps in $\mathscr{D}$, where $X$ is some object in $\mathscr{D}$, the map $\alpha_{1}: X \rightarrow T_{a}$ is a map, which when completed to a triangle $X \rightarrow T_{a} \rightarrow M^{\prime} \rightarrow$ in $\mathscr{D}$, has $M^{\prime}$ in $\mathcal{M}$, and $\alpha_{2}: X \rightarrow T_{b}$ is a map in $\mathscr{D}$. Since $\widetilde{M}=0$, we can assume that $M$ does not occur as a direct summand of $X$. Hence, $M^{\prime}$ must be in add $M$, since $\operatorname{Hom}_{\mathscr{D}}\left(T_{a}, M[1]\right)=0$. Then there is an exact sequence $\operatorname{Hom}_{\mathscr{D}}\left(T_{a}, T_{b}\right) \rightarrow \operatorname{Hom}_{\mathscr{D}}\left(X, T_{b}\right) \rightarrow \operatorname{Hom}_{\mathscr{D}}\left(M^{\prime}, T_{b}[1]\right)=0$. Thus, there is some $\beta: T_{a} \rightarrow T_{b}$ such that $\alpha_{2}=\beta \circ \alpha_{1}$. It is easily seen that the map in $\mathscr{D}^{\prime}$, represented by the pair $\left(\mathrm{id}_{T_{a}}, \beta\right)$ is equivalent to $\left(\alpha_{1}, \alpha_{2}\right)$. But the pair $\left(\operatorname{id}_{T_{a}}, \beta\right)$ represents $\tilde{\beta}$, by the definition of the localisation functor, and we have $\tilde{\beta}=\alpha$.

Now assume $\tilde{\beta}$ is an isomorphism. Then the corresponding triangle $T_{a} \rightarrow T_{b} \rightarrow$ $M^{\prime \prime} \rightarrow$ in $\mathscr{D}$ has $M^{\prime \prime}$ in $\mathcal{M}$. It is clear that $M^{\prime \prime}$ actually is in add $M$, since otherwise some $M[i]$ would be a direct summand of $T_{a}$. This means that $M^{\prime \prime}=0$, since $\operatorname{Hom}_{\mathscr{D}}\left(M, T_{a}[1]\right)=0$. Hence $\beta$ is an isomorphism.

We can now complete the main result of this section. Let $e$ be the idempotent in $\Gamma=\operatorname{End}_{\mathcal{C}}(T)^{\mathrm{op}}$, such that $\Gamma e \simeq \operatorname{Hom}_{\mathcal{C}}(T, M)$.

Theorem 2.13. With the above notation, there is a natural isomorphism $\Gamma / \Gamma e \Gamma \simeq$ $\operatorname{End}_{\mathcal{C}_{H^{\prime}}}(\widehat{T})^{\mathrm{op}}$.

The remainder of this section will be devoted to proving this theorem. Since the cluster category is defined using the functor $F=\tau^{-1}[1]$, we need to compare $\widetilde{\tau_{\mathscr{D}}^{-1}(X)}$ and $\tau_{\mathscr{D}^{\prime}}^{-1} \tilde{X}$ for an indecomposable object $X$ in $\mathscr{D}$. In general $\widetilde{\tau_{\mathscr{D}}^{-1} X} \not \tau_{\mathscr{D}^{\prime}}^{-1} \tilde{X}$, but with extra conditions on $X$, sufficient for our purposes, everything behaves nicely. We 
do not include our proof of the next lemma, since it has been generalised by Keller, with a simpler proof $[\mathrm{K}]$. Note that the existence of minimal left almost split maps is equivalent to the existence of a left Serre functor $G$ by [RV], and that $G=\tau^{-1}[-1]$.

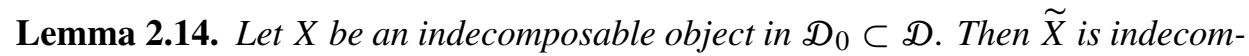
posable and $\widetilde{\tau_{\mathscr{D}}^{-1} X} \simeq \tau_{\mathscr{D}^{\prime}}^{-1} \tilde{X}$.

Let $T_{x}$ be an indecomposable direct summand in $T$, not isomorphic to $M$. Let $M_{x} \rightarrow T_{x}$ be a minimal right add $M$-approximation, and consider as before the induced triangle

$$
M_{x} \rightarrow T_{x} \rightarrow U_{x} \rightarrow
$$

in $\mathscr{D}$, where we know that $U_{x}$ is in $\mathscr{D}_{0}$ by the proof of Proposition 2.7. Thus, by applying the above lemma to each of the indecomposable direct summands of $U_{x}$, we obtain $\widetilde{\tau_{\mathscr{D}}^{-1} U_{x}} \simeq \tau_{\mathscr{D}^{\prime}}^{-1} \widetilde{U_{x}}$, and thus $\widetilde{F_{\mathscr{D}} U_{x}} \simeq F_{\mathscr{D}^{\prime}} \widetilde{U_{x}}$. It is also clear that $\widetilde{U_{x}} \simeq \widetilde{T}_{x}$.

Now, pick two (not necessarily different) indecomposable direct summands $T_{a}$ and $T_{b}$ of $\bar{T}$. Construct the triangle

$$
M_{b} \rightarrow T_{b} \rightarrow U_{b} \rightarrow
$$

as above, and apply $F$ to it, to obtain the triangle

$$
F M_{b} \rightarrow F T_{b} \rightarrow F U_{b} \rightarrow .
$$

Apply $\operatorname{Hom}_{\mathscr{D}}\left(T_{a},\right)$ to this triangle, to obtain the long exact sequence

$$
\begin{aligned}
\operatorname{Hom}_{\mathscr{D}}\left(T_{a}, F M_{b}\right) \rightarrow & \operatorname{Hom}_{\mathscr{D}}\left(T_{a}, F T_{b}\right) \\
& \rightarrow \operatorname{Hom}_{\mathscr{D}}\left(T_{a}, F U_{b}\right) \rightarrow \operatorname{Hom}_{\mathscr{D}}\left(T_{a}, F M_{b}[1]\right) .
\end{aligned}
$$

The last term vanishes, since $T_{a}$ and $M_{b}$ are modules. Since $M_{b} \rightarrow T_{b}$ is a minimal right add $M$-approximation, it follows that $F M_{b} \rightarrow F T_{b}$ is a minimal right add $F M$ approximation. We have that $\operatorname{Hom}_{\mathscr{D}}\left(T_{a}, F U_{b}\right) \simeq \operatorname{Hom}_{\mathscr{D}}\left(T_{a}, F T_{b}\right) /(F M)$, where for an object $Z$ we use the notation $\operatorname{Hom}(X, Y) /(Z)$ to denote the Hom-space modulo maps factoring through an object in add $Z$.

We claim there is an exact sequence

$$
\operatorname{Hom}_{\mathscr{D}}\left(T_{a}, F M_{b}\right) /(M) \rightarrow \operatorname{Hom}_{\mathscr{D}}\left(T_{a}, F T_{b}\right) /(M) \rightarrow \operatorname{Hom}_{\mathcal{D}}\left(T_{a}, F U_{b}\right) /(M) \rightarrow 0
$$

induced from the exact sequence (2). For this it is sufficient to show that the kernel of the second map is contained in the image of the first. Solet $\alpha \in \operatorname{Hom}_{\mathscr{D}}\left(T_{a}, F T_{b}\right) /(M)$, and assume there is a commutative diagram

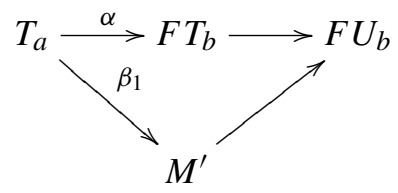


for some $M^{\prime}$ in add $M$. Since $\operatorname{Hom}_{\mathcal{D}}\left(M, F M_{b}[1]\right)=0$, there is $\beta_{2}: M^{\prime} \rightarrow F T_{b}$, such that $M^{\prime} \stackrel{\beta_{2}}{\rightarrow} F T_{b} \rightarrow F U_{b}=M^{\prime} \rightarrow F U_{b}$. In $\operatorname{Hom}_{\mathscr{D}}\left(T_{a}, F T_{b}\right) /(M)$ we have $\alpha=\alpha-\beta_{2} \beta_{1}$. By using the long exact sequence (2), we obtain that $\alpha=\alpha-\beta_{2} \beta_{1}$ factors through $F M_{b} \rightarrow F T_{b}$, so the sequence is exact. It follows from this that $\operatorname{Hom}_{\mathscr{D}}\left(T_{a}, F T_{b}\right) /(M \amalg F M) \simeq \operatorname{Hom}_{\mathscr{D}}\left(T_{a}, F U_{b}\right) /(M)$.

Let $f: M_{1} \rightarrow F U_{b}$ be a minimal right $\mathcal{M}$-approximation, and complete to a triangle $M_{1} \stackrel{f}{\rightarrow} F U_{b} \rightarrow\left(F U_{b}\right)^{\prime}$. Applying $\operatorname{Hom}_{\mathscr{D}}\left(T_{a}\right.$, $)$, we get an exact sequence

$\operatorname{Hom}_{\mathcal{D}}\left(T_{a}, M_{1}\right) \rightarrow \operatorname{Hom}_{\mathcal{D}}\left(T_{a}, F U_{b}\right) \rightarrow \operatorname{Hom}_{\mathcal{D}}\left(T_{a},\left(F U_{b}\right)^{\prime}\right) \rightarrow \operatorname{Hom}_{\mathcal{D}}\left(T_{a}, M_{1}[1]\right)$.

Since $U_{b}$ is in degree 0 or 1 , then $F U_{b}$ is in degree 1,2 or 3 , so $M_{1}$ is in degree $0,1,2$ or 3 . Hence the indecomposable direct summands of $M_{1}[1]$ are in degree at least 1 , so that $\operatorname{Hom}_{\mathscr{D}}\left(T_{a}, M_{1}[1]\right)=0$. Since a map $h: T_{a} \rightarrow F U_{b}$ factors through an object in $\mathcal{M}$ if and only if it factors through the minimal right $\mathcal{M}$-approximation of $F U_{b}$, we get the isomorphism

$$
\operatorname{Hom}_{\mathscr{D}}\left(T_{a}, F_{\mathscr{D}} U_{b}\right) /(M) \simeq \operatorname{Hom}_{\mathscr{D}}\left(T_{a},\left(F_{\mathscr{D}} U_{b}\right)^{\prime}\right) .
$$

We get that this is isomorphic to $\operatorname{Hom}_{\mathscr{D}^{\prime}}\left(\widetilde{T_{a}}, \widetilde{F_{\mathscr{D}} U_{b}}\right)$, since $\left(F_{\mathscr{D}} U_{b}\right)^{\prime}$ is in $\mathscr{D}_{0}$. By Lemma 2.14 this is isomorphic to $\operatorname{Hom}_{\mathscr{D}^{\prime}}\left(\widetilde{T}_{a}, F_{D^{\prime}} \widetilde{T}_{b}\right)$. We thus obtain that

$$
\operatorname{Hom}_{\mathscr{D}}\left(T_{a}, F_{\mathscr{D}} T_{b}\right) /(M \amalg F M) \simeq \operatorname{Hom}_{D^{\prime}}\left(\widetilde{T}_{a}, F_{D^{\prime}} \widetilde{T}_{b}\right) .
$$

We have $\operatorname{Hom}_{\mathscr{D}}\left(T_{a}, T_{b}\right) /(M \amalg F M) \simeq \operatorname{Hom}_{\mathscr{D}}\left(T_{a}, T_{b}\right) /(M)$. Consider again the triangle $M_{b} \stackrel{f_{b}}{\rightarrow} T_{b} \rightarrow U_{b}$ in $\mathscr{D}$, where $f_{b}: M_{b} \rightarrow T_{b}$ is a minimal right $\mathcal{M}$-approximation. Applying $\operatorname{Hom}_{\mathscr{D}}\left(T_{a}\right.$, ) gives an exact sequence

$$
\operatorname{Hom}_{\mathscr{D}}\left(T_{a}, M_{b}\right) \rightarrow \operatorname{Hom}_{\mathscr{D}}\left(T_{a}, T_{b}\right) \rightarrow \operatorname{Hom}_{\mathscr{D}}\left(T_{a}, U_{b}\right) \rightarrow \operatorname{Hom}_{\mathscr{D}}\left(T_{a}, M_{b}[1]\right) .
$$

Since $M_{b}$ is a module, we have $\operatorname{Hom}_{\mathscr{D}}\left(T_{a}, M_{b}[1]\right)=0$, and hence $\operatorname{Hom}_{\mathscr{D}}\left(T_{a}, T_{b}\right) /(M) \simeq \operatorname{Hom}_{\mathscr{D}}\left(T_{a}, U_{b}\right)$, which is isomorphic to $\operatorname{Hom}_{D^{\prime}}\left(\widetilde{T}_{a}, \widetilde{U}_{b}\right)$ by Proposition 2.2. We obtain that:

$$
\operatorname{Hom}_{\mathscr{D}}\left(T_{a}, T_{b}\right) /(M \amalg F M) \simeq \operatorname{Hom}_{D^{\prime}}\left(\widetilde{T_{a}}, \widetilde{T_{b}}\right) .
$$

Hence $\Gamma / \Gamma e \Gamma=\operatorname{Hom}_{\mathscr{D}}(T, T) \operatorname{HHom}_{\mathscr{D}}(T, F T) /(M \amalg F M) \simeq \operatorname{Hom}_{\mathcal{D}^{\prime}}(\widetilde{T}, \widetilde{T}) \amalg$ $\operatorname{Hom}_{D^{\prime}}\left(\widetilde{T}, F_{D^{\prime}} \widetilde{T}\right)$ as vector spaces. It is straightforward to check that the map is also a ring map. Theorem 2.13 is proved.

2.5. Comparison with tilted algebras. We give an example showing that a result similar to Theorem 2.13 does not hold for tilted algebras. We would like to thank Dieter Happel for providing us with this example. There is a tilting module for 
the path algebra of a Dynkin quiver of type $D_{5}$, such that the corresponding tilted algebra $\Lambda$ has the quiver

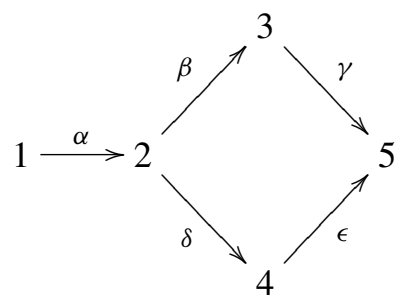

with relations $\alpha \beta=\beta \gamma-\delta \epsilon=0$. If we let $e_{4}$ be the primitive idempotent corresponding to vertex 4 , then $\Lambda / \Lambda e_{4} \Lambda$ is not tilted, since it has global dimension three.

It is well known that the endomorphism-ring of a partial tilting module is a tilted algebra. However, a similar result does not hold for cluster-tilted algebras. An example of this is the path algebra of an oriented 4-cycle, modulo the cube of its radical. This is a cluster-tilted algebra of type $D_{4}$.

\section{Cluster-tilted algebras of rank at most 2}

In this section we apply the main result of the previous section to show that (oriented) cycles in the quiver of a cluster-tilted algebra have length at least three. For the rest of the paper, the field $K$ is assumed to be algebraically closed. Let $T_{1} \amalg T_{2} \amalg \cdots \amalg T_{n}$ be a tilting object in the cluster category $\mathcal{C}$. We denote by $\delta_{k}(T)$ the tilting object $T^{\prime}$ obtained by exchanging $T_{k}$ with the second complement of $T_{1} \amalg \cdots \amalg T_{k-1} \amalg$ $T_{k+1} \amalg \cdots U T_{n}$. Let $\Gamma=\operatorname{End}_{\mathcal{C}}(T)^{\text {op }}$ and $\Gamma^{\prime}=\operatorname{End}_{\mathcal{C}}\left(T^{\prime}\right)^{\text {op }}$ be the corresponding cluster-tilted algebras. Passing from $\Gamma$ to $\Gamma^{\prime}$ depends on the choice of tilting object $T$. But we still write $\bar{\delta}_{k}(\Gamma)=\Gamma^{\prime}$, when either it is clear from the context which tilting object $T$ gives rise to $\Gamma$, or when this is irrelevant. We also say that $\Gamma^{\prime}$ is obtained from $\Gamma$ by mutation at $k$.

From [BMRRT] we know that all tilting objects in $\mathcal{C}_{H}$ can be obtained from performing a finite number of operations $\delta_{k}$ to $H$, where $H$ is the hereditary algebra considered as a tilting object in $\mathcal{C}_{H}$.

If $k$ is a source or a sink in the quiver of a hereditary algebra, then mutation at $k$ coincides with so-called APR-tilting [APR] (see [BMR]), and the quiver of the mutated algebra $\bar{\delta}_{k}(H)$ is obtained by reversing all arrows ending or starting in $k$.

Lemma 3.1. The cluster-tilted algebras of rank at most 2 are hereditary.

Proof. This follows from the fact that any cluster-tilted algebra can be obtained by starting with a hereditary algebra, and performing a finite number of mutations. If we 
start with a hereditary algebra $H$ of rank at most 2, the algebra obtained by mutating at one of the vertices is isomorphic to $H$.

Proposition 3.2. The quiver of a cluster-tilted algebra has no loops and no cycles of length 2.

Proof. This follows directly from combining Lemma 3.1 with Theorem 2.13.

Note that the assertion of no loops was proven in [BMRRT, Cor. 6.15], while the second assertion was first proven by Gordana Todorov in case of finite representation type.

\section{Cluster-tilted algebras of rank 3}

In this section we specialize to connected hereditary algebras of rank 3 , and the clustertilted algebras obtained from them. We describe the possible quivers, and give some information on the relation-spaces. Later, this will be used to show our main result for algebras of rank 3. In the proof of our main theorem, we use Theorem 2.13 to reduce to the case of rank 3 . For hereditary algebras of finite representation type, there is up to derived equivalence only one connected algebra of rank 3 , and thus up to equivalence only one cluster category $\mathcal{C}$. In this case the technically involved results of this section reduce to just checking one case: The only cluster-tilted algebra of rank 3 which is not hereditary is given by a quiver which is a cycle of length 3 , and with the relations that the composition of any two arrows is zero.

4.1. The quivers. We consider quivers of the form

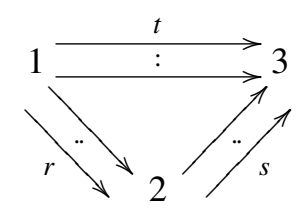

where $r>0, s>0$ and $t \geq 0$ denote the number of arrows as indicated in the above figure. For short, we denote such a quiver by $Q_{r s t}$.

Up to derived equivalence, all connected finite dimensional hereditary algebras of rank 3 have a quiver given as above. We first note that factors of path-algebras of such quivers by non-zero admissible ideals are never cluster-tilted. We omit our original proof of this fact since it is a consequence of the more general (recently proven) fact from $[\mathrm{KR}]$ that any cluster-tilted algebra is either hereditary or of infinite global dimension. Hence, since $Q_{r s t}$ has no oriented cycles, it follows that if it is the quiver of a cluster-tilted algebra, there can be no proper relations. So we have the following. 
Lemma 4.1. If $\Gamma$ is a cluster-tilted algebra with quiver of type $Q_{r s t}$, then $\Gamma$ is hereditary.

This has the following consequence.

Corollary 4.2. The quiver of a non-hereditary connected cluster-tilted algebra of rank 3 is of the form

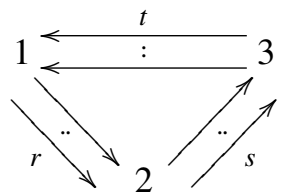

with $r, s, t>0$.

Proof. Combine Lemma 4.1 with Proposition 3.2.

In view of this we refer to the cluster-tilted algebras of rank 3 which are nonhereditary as cyclic cluster-tilted algebras.

4.2. The relations. We first show that relations are homogeneous.

Proposition 4.3. Let $\Gamma$ be a cluster-tilted algebra of rank 3 with Jacobson radical $\underline{r}$. Then $\underline{r}^{6}=0$, and the relations are homogeneous.

Proof. Without loss of generality we can assume that there is a tilting module $T=$ $X \amalg Y \amalg Z$ for a hereditary algebra $H$, such that $\Gamma=\operatorname{End}_{\mathcal{C}_{H}}(T)^{\mathrm{op}}$. form

Using Corollary 4.2 it is clear that we can assume that the quiver of $\Gamma$ has the

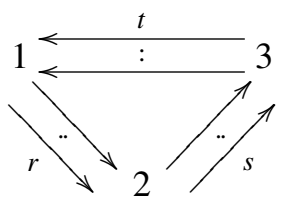

with $r, s, t>0$.

Let $\Lambda=\operatorname{End}_{H}(T)^{\text {op }}$ be the corresponding tilted algebra. There are no cycles in the quiver of a tilted algebra. We can therefore assume that there is a sink in the quiver of $\Lambda$, and we assume that this vertex corresponds to $Z$, that is, $\operatorname{Hom}_{H}(Z, X \amalg Y)=0$. We assume that $X, Y, Z$ correspond to the vertices $1,2,3$, respectively. If $\hat{h}$ is a non-zero map in $\operatorname{Irr}_{\text {add }} T(Z, X)$, it must be of degree 1 , that is, the lifting $h$ is in $\operatorname{Hom}_{\mathcal{D}}(Z, F X)$. Since this holds for all maps in $\operatorname{Irr}_{\text {add }} T(Z, X)$, any composition of 6 arrows will correspond to a map of degree $\geq 2$ from an indecomposable to itself, and therefore must be the zero-map. This follows from the fact that for any indecomposable module $M$, we have $\operatorname{Hom}_{\mathscr{D}}\left(M, F^{2} M\right)=0$. This gives $\underline{r}^{6}=0$. 
We can assume that at least one of the arrows (irreducible maps) $X \rightarrow Y$ and at least one of the arrows $Y \rightarrow Z$ are of degree 0 . Otherwise, the tilted algebra $\Lambda$ would not be connected.

Now let $\hat{g}$ be a map in $\operatorname{Irr}_{\text {add }} T(Y, Z)$. We want to show that it must be of degree 0 . Since $X \amalg Y$ is an almost complete tilting object in $\mathcal{C}_{H}$, there are exactly two complements. Denote as usual the second one by $Z^{*}$. The complement $Z^{*}$ is either the image of a module or the image of an object of the form $I[-1]$ for an injective indecomposable module $I$. Furthermore, there is a triangle in $\mathcal{C}$

$$
Z^{*} \rightarrow Y^{s} \rightarrow Z \rightarrow
$$

for some $s \geq 0$, which can be lifted to a triangle

$$
F^{i} Z^{*} \stackrel{\left(\begin{array}{l}
\alpha_{1} \\
\alpha_{2}
\end{array}\right)}{\rightarrow} Y^{s_{1}} \amalg\left(F^{-1} Y\right)^{s_{2}} \rightarrow Z \rightarrow
$$

in $\mathscr{D}$ for some integer $i$ and with $s=s_{1}+s_{2}$. We need to show that $s_{2}=0$. It is sufficient to show that the map $\alpha_{2}=0$. We have $s_{1} \neq 0$, and thus by minimality $\alpha_{1} \neq 0$. It is clear that if also $\alpha_{2} \neq 0$, then $i=0$ or $i=-1$.

Assume first $Z^{*} \simeq I[-1]$, then

$$
\operatorname{Hom}_{\mathscr{D}}\left(I[-1], F^{-1} Y\right)=\operatorname{Hom}_{\mathscr{D}}(I, \tau Y)=0,
$$

so $i=0$ gives $\alpha_{2}=0$. On the other hand, it is clear that $i=-1$ gives $\alpha_{1}=0$.

Assume now that $Z^{*}$ is the image of a module. Then there is an exact sequence of modules

$$
0 \rightarrow Z^{*} \rightarrow Y^{v} \rightarrow Z \rightarrow 0
$$

and since $\operatorname{dim}_{k} \operatorname{Hom}_{\mathcal{C}}\left(Z, Z^{*}[1]\right)=1$ (by [BMRRT]), it follows that the triangle (3) is induced by this sequence, and thus $s_{1}=v$ and $s_{2}=0$.

Now we show that all the irreducible maps $X \rightarrow Y$ in $\mathcal{C}_{H}$ are of degree 0 . For this, consider the almost complete tilting object $X \amalg Z$ in $C_{H}$, with complements $Y$ and $Y^{*}$. Consider the triangle

$$
Y^{*} \rightarrow X^{r} \rightarrow Y \rightarrow
$$

and the preimage in $\mathscr{D}$,

$$
F^{i} Y^{*} \stackrel{\left(\begin{array}{l}
\beta_{1} \\
\beta_{2}
\end{array}\right)}{\rightarrow} X^{r_{1}} \amalg\left(F^{-1} X\right)^{r_{2}} \rightarrow Y \rightarrow .
$$

We need to show that $r_{2}=0$. The case where $Y^{*} \simeq I[-1]$ is completely similar as for irreducible maps $Y \rightarrow Z$. In case $Y^{*}$ is the image of a module, it is now more complicated since we have two possibilities. Either there is an exact sequence in $\bmod H$ of the form

$$
0 \rightarrow Y^{*} \rightarrow X^{a} \rightarrow Y \rightarrow 0
$$


or there is an exact sequence of the form

$$
0 \rightarrow Y \rightarrow Z^{b} \rightarrow Y^{*} \rightarrow 0
$$

If we are in the first case, we can use the same argument as for irreducible maps $Y \rightarrow Z$. If we are in the second case, note that $\operatorname{Hom}_{H}\left(Y^{*}, X\right)=0$, since $\operatorname{Hom}_{H}(Z, X)=0$. Thus, either $\beta_{1}=0$ or $\beta_{2}=0$ in our triangle. This completes the proof that all irreducible maps $X \rightarrow Y$ are induced by module maps, and thus are of degree 0 .

Given that $\underline{r}^{6}=0$, the only possibility for a non-homogeneous relation must involve maps in $\underline{r}^{2} \backslash \underline{r}^{3}$ and maps in $\underline{r}^{5}$. But, by our description of irreducible maps, this is not possible, because it would involve a relation between maps of different degrees.

Fix a cyclic cluster-tilted algebra of rank 3, and fix a vertex $k$. Let $\alpha$ be an arrow ending in $k$, and $\beta$ an arrow starting in $k$. If $\beta \alpha=0$, as an element of the algebra, for any choice of $\alpha$ and $\beta$, then we call $k$ a zero vertex.

Proposition 4.4. Let $\Gamma$ be a cyclic cluster-tilted algebra, and fix a vertex $k$. Then $k$ is a zero-vertex if and only if $\bar{\delta}_{k}(\Gamma)$ is hereditary.

Proof. We assume the quiver of $\Gamma$ is

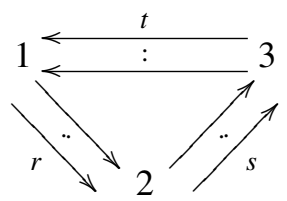

Let $\Gamma=\operatorname{End}_{\mathcal{C}}(T)^{\mathrm{op}}$, and let $T_{i}$ be the direct summand of $T$ corresponding to the vertex $i$. Assume that 2 is a zero-vertex. Then it is clear that $\operatorname{Hom}_{\mathcal{C}}\left(T_{1}, T_{3}\right)=0$, so the quiver of $\bar{\delta}_{2}(\Gamma)$ must be

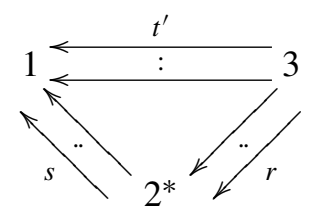

with $t^{\prime} \geq 0$. Now $\bar{\delta}_{2}(\Gamma)$ is hereditary, by Lemma 4.1 .

Conversely, assume $\bar{\delta}_{k}(\Gamma)$ is hereditary. The quiver of $\bar{\delta}_{k}(\Gamma)$ must be as above, with $t^{\prime} \geq 0$. This means $\operatorname{Hom}_{\mathcal{C}}\left(T_{1}, T_{3}\right)=0$, so 2 is a zero-vertex. 
4.3. Kerner's Theorem. The following result by Kerner [Ke] turns out to be crucial for the proof of the main theorem of this section. There is a more general version of this theorem in [Ke]. We include a proof, for the convenience of the reader. This proof is also due to Kerner, and we thank him for providing us with it.

Theorem 4.5. Let $X, Y$ be regular indecomposable modules over a wild hereditary algebra $H$ of rank 3. If $\operatorname{Hom}_{H}(X, \tau Y)=0$, then also $\operatorname{Hom}_{H}\left(X, \tau^{-1} Y\right)=0$.

Proof. We first prove the following.

Lemma 4.6. Let $U$ be an indecomposable regular module over a wild hereditary algebra of rank 3. Then $\operatorname{Hom}_{H}\left(U, \tau^{2} U\right) \neq 0$.

Proof. Assume first that $\operatorname{Ext}_{H}^{1}(U, U) \neq 0$. Then also $\operatorname{Hom}_{H}(U, \tau U) \neq 0$ by the AR-formula. Assume now $\operatorname{Hom}_{H}\left(U, \tau^{2} U\right)=0$. Then also $\operatorname{Ext}_{H}^{1}(\tau U, U)=0$ and, by the Happel-Ringel lemma [HR], a non-zero map $f: U \rightarrow \tau U$ is either surjective or injective. In either case, $g=\tau(f) \circ f: U \rightarrow \tau^{2} U$ is non-zero. This contradicts $\operatorname{Hom}_{H}\left(U, \tau^{2} U\right)=0$.

Now assume $\operatorname{Ext}_{H}^{1}(U, U)=0$. Then by [Ho], $U$ is quasi-simple. Thus, there is an almost split sequence $0 \rightarrow \tau U \rightarrow V \rightarrow U \rightarrow 0$, where $V$ is indecomposable, and by [Ke2] we have $\operatorname{End}_{H}(V) \simeq K$, while $\operatorname{Ext}_{H}^{1}(V, V) \neq 0$. Applying $\operatorname{Hom}_{H}(U$,$) to$ the almost split sequence, we obtain the exact sequence

$$
\operatorname{Hom}_{H}(U, \tau U) \rightarrow \operatorname{Hom}_{H}(U, V) \rightarrow \operatorname{Hom}_{H}(U, U) \rightarrow \operatorname{Ext}_{H}^{1}(U, \tau U) .
$$

Since $\operatorname{Hom}_{H}(U, U) \rightarrow \operatorname{Ext}_{H}^{1}(U, \tau U)$ is an isomorphism and $\operatorname{Hom}_{H}(U, \tau U)=0$, we have that also $\operatorname{Hom}_{H}(U, V)=0$. The long exact sequence obtained by applying $\operatorname{Hom}_{H}(, \tau U)$ to the almost split sequence, gives $\operatorname{Hom}_{H}(V, \tau U)=0$. Now, this gives $\operatorname{Hom}_{H}\left(V, \tau^{2} U\right) \neq 0$, since there is an exact sequence

$$
0 \rightarrow \operatorname{Hom}_{H}\left(V, \tau^{2} U\right) \rightarrow \operatorname{Hom}_{H}(V, \tau V) \rightarrow \operatorname{Hom}_{H}(V, \tau U)
$$

and the last term is zero. There is also the long exact sequence

$$
0 \rightarrow \operatorname{Hom}_{H}\left(U, \tau^{2} U\right) \rightarrow \operatorname{Hom}_{H}\left(V, \tau^{2} U\right) \rightarrow \operatorname{Hom}_{H}\left(\tau U, \tau^{2} U\right)
$$

where the last term is zero. This proves $\operatorname{Hom}_{H}\left(U, \tau^{2} U\right) \neq 0$.

Let us now complete the proof of the theorem. Let $X, Y$ be regular indecomposable modules. It suffices to show that $\operatorname{Hom}_{H}(X, Y) \neq 0$ implies $\operatorname{Hom}_{H}\left(X, \tau^{2} Y\right) \neq 0$. Let $z: X \rightarrow Y$ be a non-zero map. Then we can assume there is an indecomposable regular module $U$, such that $z$ factors as $X \stackrel{p}{\rightarrow} U \stackrel{i}{\rightarrow} Y$, where $p$ is surjective and $i$ is injective. Also $\tau^{2} i: \tau^{2} U \rightarrow \tau^{2} Y$ is injective. By Lemma 4.6, there is a non-zero map $f: U \rightarrow \tau^{2} U$. The composition $\tau^{2} i \circ f \circ p$ is non-zero. This completes the proof of the theorem. 
4.4. The dimensions of relation-spaces. Let $H$ be a connected hereditary algebra of rank 3. The following notation is used for the rest of this section. Let $\bar{T}$ be an almost complete tilting object with complements $M$ and $M^{*}$, and assume there are triangles as in Theorem 1.2. Let $T=\bar{T} \amalg M$ and $T^{\prime}=\bar{T} \amalg M^{*}$ and let $\Gamma=\operatorname{End}_{\mathcal{C}}(T)^{\text {op }}$ and $\Gamma^{\prime}=\operatorname{End}_{\mathcal{C}}\left(T^{\prime}\right)^{\text {op }}$. By now, we know that the quiver of $\Gamma$ is either

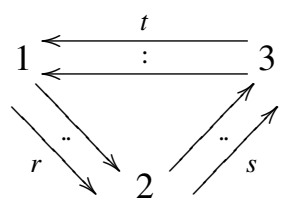

with $r, s, t>0$ or the quiver $Q_{r s t}$

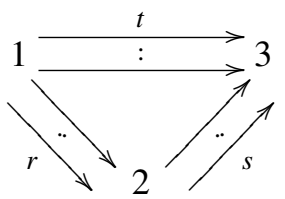

with $r, s>0$ and $t \geq 0$. We let $M$ correspond to vertex 2. Then $\bar{T}=T_{B} \amalg T_{B^{\prime}}$ where $T_{B}$ corresponds to the vertex 1 and $T_{B^{\prime}}$ to 3 . It is then clear that $B=\left(T_{B}\right)^{r}$ and $B^{\prime}=\left(T_{B^{\prime}}\right)^{s}$. We label the vertices with the corresponding modules, then the arrows represent irreducible maps in add $T$.

We let $I$ denote the ideal such that $\Gamma \simeq K Q / I$. In case $\Gamma$ is cyclic, we say that $\Gamma$ is balanced at the vertex 2 if

$$
\operatorname{dim}\left(\left(\operatorname{Irr}\left(T_{B}, M\right) \otimes_{K} \operatorname{Irr}\left(M, T_{B^{\prime}}\right) \cap I\right)=t .\right.
$$

We will show that any vertex of a cyclic cluster-tilted algebra is either balanced or a zero-vertex. We first discuss the algebras obtained by mutating hereditary algebras.

Lemma 4.7. Let $H$ be a hereditary algebra with quiver $Q_{r s t}$ where $r, s>0$ and $t \geq 0$. Then the following hold.

(a) The cluster-tilted algebra $\Gamma^{\prime}=\bar{\delta}_{2}(H)$ is balanced at the vertices 1 and 3 .

(b) The new vertex $2 *$ is a zero-vertex.

(c) The quiver of $\Gamma^{\prime}$ is

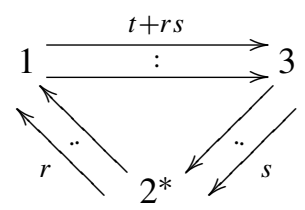


Proof. Part (b) and (c) follow directly from Lemma 4.1 and Proposition 4.4. Let $P_{i}$ be the indecomposable projective $H$-module corresponding to vertex $i$, and $S_{i}$ the simple $H$-module $P_{i} / \underline{\mathrm{r}} P_{i}$. Then $P_{1}=S_{1}$ is simple. Consider $P_{1} \amalg P_{3}$ as an almost complete tilting object. There is an exact sequence

$$
0 \rightarrow P_{2} \rightarrow\left(P_{1}\right)^{s} \rightarrow P_{2}^{*} \rightarrow 0,
$$

such that the induced triangle in $\mathcal{C}$ is the exchange-triangle of Theorem 1.2. Let $T^{\prime}=P_{1} \amalg P_{2}^{*} \amalg P_{3}$. Using the definition of $\tau$, one can show that $S_{2}=\tau P_{2}^{*}$, and thus $\operatorname{Hom}_{D}\left(P_{2}^{*}, P_{3}\right)=0$. Since $2^{*}$ is a zero-vertex, $\operatorname{Irr}_{\text {add } T^{\prime}}\left(P_{1}, P_{3}\right)=\operatorname{Hom}_{H}\left(P_{1}, P_{3}\right)$, with dimension $r s+t$. We want to compute $\operatorname{Irr}_{\text {add }} T^{\prime}\left(P_{2}^{*}, P_{1}\right) \otimes_{\Gamma^{\prime}} \operatorname{Irr}_{\text {add }} T^{\prime}\left(P_{1}, P_{3}\right)=$ $\operatorname{Hom}_{\mathcal{C}}\left(P_{2}^{*}, P_{3}\right) \simeq \operatorname{Hom}_{\mathscr{D}}\left(F^{-1} P_{2}^{*}, P_{3}\right)$. We have

$$
\operatorname{Hom}_{\mathscr{D}}\left(F^{-1} P_{2}^{*}, P_{3}\right)=\operatorname{Hom}_{\mathscr{D}}\left(\tau P_{2}^{*}[-1], P_{3}\right)=\operatorname{Ext}_{H}^{1}\left(S_{2}, P_{3}\right) .
$$

There is an exact sequence

$$
0 \rightarrow\left(P_{1}\right)^{r} \rightarrow P_{2} \rightarrow S_{2} \rightarrow 0 .
$$

Apply $\operatorname{Hom}_{H}\left(, P_{3}\right)$ to it, to obtain the long exact sequence

$$
\begin{aligned}
0 \rightarrow & \operatorname{Hom}_{H}\left(S_{2}, P_{3}\right) \rightarrow \operatorname{Hom}_{H}\left(P_{2}, P_{3}\right) \\
& \rightarrow \operatorname{Hom}_{H}\left(\left(P_{1}\right)^{r}, P_{3}\right) \rightarrow \operatorname{Ext}_{H}^{1}\left(S_{2}, P_{3}\right) \rightarrow 0 .
\end{aligned}
$$

Since $\operatorname{dim} \operatorname{Hom}_{H}\left(P_{2}, P_{3}\right)=s$, and $\operatorname{dim} \operatorname{Hom}_{H}\left(\left(P_{1}\right)^{r}, P_{3}\right)=(r s+t) r$, it follows that $\operatorname{dim}\left(\operatorname{Irr}_{\text {add } T^{\prime}}\left(P_{2}^{*}, P_{1}\right) \otimes_{\Gamma^{\prime}} \operatorname{Irr}_{\text {add } T^{\prime}}\left(P_{1}, P_{3}\right)\right)=r(r s+t)-s$, and therefore $\operatorname{dim}\left(\operatorname{Irr}_{\text {add } T^{\prime}}\left(P_{2}^{*}, P_{1}\right) \otimes_{K} \operatorname{Irr}_{\text {add } T^{\prime}}\left(P_{1}, P_{3}\right) \cap I\right)=s$, and $\Gamma^{\prime}$ is balanced at 3 .

Now apply $\operatorname{Hom}_{H}\left(P_{1}, \quad\right)$ to the exact sequence $0 \rightarrow P_{2} \rightarrow\left(P_{3}\right)^{S} \rightarrow P_{2}^{*} \rightarrow 0$ to obtain the exact sequence

$$
0 \rightarrow \operatorname{Hom}_{H}\left(P_{1}, P_{2}\right) \rightarrow \operatorname{Hom}_{H}\left(P_{1}, P_{3}^{s}\right) \rightarrow \operatorname{Hom}_{H}\left(P_{1}, P_{2}^{*}\right) \rightarrow 0 .
$$

Since $\operatorname{dim} \operatorname{Hom}_{H}\left(P_{1}, P_{2}\right)=r$ and $\operatorname{dim} \operatorname{Hom}_{H}\left(P_{1}, P_{3}^{s}\right)=(r s+t) s$, we have $\operatorname{dim}\left(\operatorname{Irr}_{\text {add } T^{\prime}}\left(P_{1}, P_{3}\right) \otimes_{\Gamma^{\prime}} \operatorname{Irr}_{\text {add } T^{\prime}}\left(P_{3}, P_{2}^{*}\right)\right)=\operatorname{dim}_{\operatorname{Hom}}\left(P_{1}, P_{2}^{*}\right)=(r s+t) s-r$. This means $\operatorname{dim}\left(\operatorname{Irr}_{\text {add }} T^{\prime}\left(P_{1}, P_{3}\right) \otimes_{K} \operatorname{Irr}_{\text {add }} T^{\prime}\left(P_{3}, P_{2}^{*}\right) \cap I\right)=r$, and $\Gamma^{\prime}$ is balanced also at 1 .

Proposition 4.8. Let $\Gamma$ be a non-hereditary cluster-tilted algebra with quiver

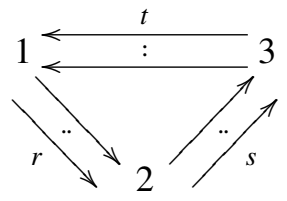


(a) If $\Gamma$ is balanced at the vertex 2 , then $\Gamma^{\prime}=\bar{\delta}_{2}(\Gamma)$ is non-hereditary, and thus cyclic, with quiver

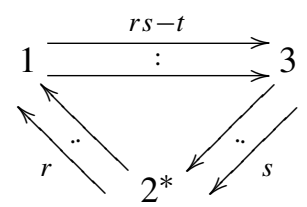

It is balanced at the new vertex $2^{*}$. Each of the other vertices of $\Gamma^{\prime}$ is either balanced or a zero-vertex.

(b) If $\Gamma$ has a zero-vertex at 2 , then $\bar{\delta}_{2}(\Gamma)$ is hereditary with quiver

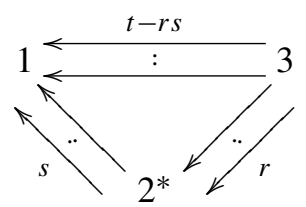

Proof. Part (b) follows from Propositions 4.3 and 4.4.

To prove part (a), we adopt our earlier notation and conventions. Especially, $\Gamma=\operatorname{End}_{\mathcal{C}}\left(T_{B} \amalg T_{B^{\prime}} \amalg M\right)^{\mathrm{op}}$, and we have the quiver

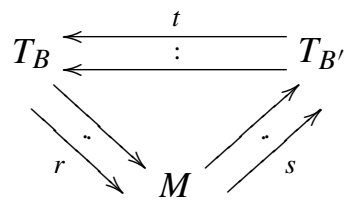

The quiver of the mutated algebra $\bar{\delta}_{2}(\Gamma)=\operatorname{End}_{\mathcal{C}}\left(T_{B} \amalg T_{B^{\prime}} \amalg M^{*}\right)^{\text {op }}$ is

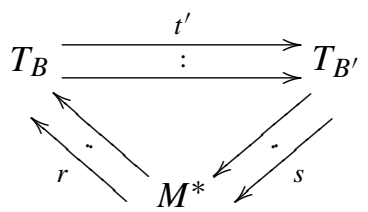

Using that $\Gamma$ is balanced at 2, and Proposition 4.3, it follows that $t^{\prime}=r s-t$. Also by assumption, $M$ does not correspond to a zero-vertex, so there is at least one non-zero composition $T_{B} \rightarrow M \rightarrow T_{B^{\prime}}$. Therefore $r s-t>0$.

We have $\operatorname{dim}\left(\operatorname{Irr}_{\text {add } T^{\prime}}\left(T_{B^{\prime}}, M^{*}\right) \otimes_{\Gamma^{\prime}} \operatorname{Irr}_{\text {add } T^{\prime}}\left(M^{*}, T_{B}\right)\right)=\operatorname{dim} \operatorname{Irr}_{\text {add } T}\left(T_{B^{\prime}}, T_{B}\right)+$ $\operatorname{dim}\left(\operatorname{Irr}_{\text {add } T}\left(T_{B^{\prime}}, M\right) \otimes_{\Gamma^{\prime}} \operatorname{Irr}_{\text {add } T}\left(M, T_{B}\right)\right)=t+0$. Hence $\operatorname{dim}\left(\operatorname{Irr}_{\text {add }} T^{\prime}\left(T_{B^{\prime}}, M^{*}\right) \otimes_{K}\right.$ $\left.\operatorname{Irr}_{\text {add } T^{\prime}}\left(M^{*}, T_{B}\right) \cap I\right)=r s-t$, so $\Gamma^{\prime}$ is balanced at $2^{*}$.

We now proceed to show that for each of the vertices 1 and $3, \Gamma^{\prime}$ is either balanced, or a zero-vertex. We assume $T_{B^{\prime}}$ is not a zero-vertex in $\Gamma^{\prime}$. 
The tilted algebra $\Lambda=\operatorname{End}_{H}\left(T_{B} \amalg T_{B^{\prime}} \amalg M\right)^{\text {op }}$ has a unique sink. There is an induced total ordering on the triple $T_{B}, T_{B^{\prime}}, M$, where the last element in the ordering corresponds to the sink. Also, by considering the preimage of $M^{*}$ in the standard domain of $\mathscr{D}$, the ordering can be extended to the quadruple $B^{\prime}, M^{*}, B, M$. Note that we get the following four possible orderings

$$
\begin{aligned}
& -\left(M, T_{B^{\prime}}, M^{*}, T_{B}\right), \\
& -\left(T_{B^{\prime}}, M^{*}, T_{B}, M\right), \\
& -\left(M^{*}, T_{B}, M, T_{B^{\prime}}\right), \\
& -\left(T_{B}, M, T_{B^{\prime}}, M^{*}\right) .
\end{aligned}
$$

First we show the claim for the vertex corresponding to $T_{B^{\prime}}$.

Lemma 4.9. Assume that $T_{B^{\prime}}$ does not correspond to a zero-vertex in $\Gamma^{\prime}$ and that $M^{*}$ is before $T_{B}$ in the above ordering. Then $\operatorname{Hom}_{D}\left(T_{B}, \tau^{-1} M^{*}\right)=0$.

Proof. Since $\bar{\delta}_{2}(\Gamma)$ is not hereditary, we have $\operatorname{Hom}_{H}\left(M^{*}, T_{B}\right) \neq 0$. Assume now that $\operatorname{Hom}_{H}\left(T_{B}, \tau^{-1} M^{*}\right) \neq 0$. Assume first that $T_{B}$ is regular, then $M^{*}$ is also regular. In case $H$ is tame, then there are at most two exceptional modules which are regular. This follows from the fact that $H$ has three simples. But in case there are two exceptional modules which are regular, there is an extension between them. This gives a contradiction. In case $H$ is wild we can apply Kerner's Theorem, which says that $\operatorname{Hom}_{H}\left(T_{B}, \tau M^{*}\right) \neq 0$. We have a contradiction, since $\operatorname{Hom}_{H}\left(T_{B}, \tau M^{*}\right) \simeq$ $D \operatorname{Ext}_{H}^{1}\left(M^{*}, T_{B}\right)=0$.

If $B$ is a preprojective or a preinjective module, then $\operatorname{Hom}_{H}\left(M^{*}, T_{B}\right) \neq 0$ and $\operatorname{Hom}_{H}\left(T_{B}, \tau^{-1} M^{*}\right) \neq 0$ implies that the map $M^{*} \rightarrow T_{B}$ is irreducible in the modulecategory. Thus $M^{*} \amalg T_{B}$ can be complemented to a tilting module with hereditary endomorphism ring. We have seen that the mutated algebra $\bar{\delta}_{2}(\Gamma)$ is by assumption not hereditary, i.e., $T_{B^{\prime}}$ must correspond to a zero-vertex, so we have a contradiction to $\operatorname{Hom}_{H}\left(B, \tau^{-1} M^{*}\right) \neq 0$ also for $T_{B}$ being preprojective or preinjective.

Now, let $M \rightarrow B^{\prime} \rightarrow M^{*} \rightarrow$ be the usual triangle. We recall that $\bar{T}=T_{B} \amalg T_{B^{\prime}}$. Let $\widetilde{\operatorname{Hom}}\left(T_{B}, M\right)=\operatorname{Irr}_{\text {add } T}\left(T_{B}, M\right)$, let $\widetilde{\operatorname{Hom}}\left(T_{B}, B^{\prime}\right)=\operatorname{Irr}_{\text {add }}\left(T_{B}, B^{\prime}\right)$ and let $\widetilde{\operatorname{Hom}}\left(T_{B}, M^{*}\right)=\operatorname{Irr}_{\text {add } T^{\prime}}\left(T_{B}, M^{*}\right)$. Then we claim that there is an exact sequence

$$
0 \rightarrow \widetilde{\operatorname{Hom}}\left(T_{B}, M\right) \stackrel{\alpha}{\rightarrow} \widetilde{\operatorname{Hom}}\left(T_{B}, B^{\prime}\right) \rightarrow \widetilde{\operatorname{Hom}}\left(T_{B}, M^{*}\right) \rightarrow 0 .
$$

It is clear from Proposition 4.3, and the fact that

$$
\operatorname{Hom}_{\mathscr{D}}\left(T_{B}, M\right) \rightarrow \operatorname{Hom}_{\mathcal{D}}\left(T_{B}, B^{\prime}\right) \rightarrow \operatorname{Hom}_{\mathcal{D}}\left(T_{B}, M^{*}\right) \rightarrow 0
$$

is exact, that we only need to show that the map $\alpha$ is a monomorphism. We first assume $M^{*}$ is a module. To prove the claim for this case, we consider the four 
orderings on the quadruple $\left\{M, B^{\prime}, M^{*}, B\right\}$. For each case we show that a map in $\operatorname{Irr}_{\text {add } T}\left(T_{B}, M\right)$ cannot factor via $M^{*}[-1]$ in $\mathcal{C}$.

$\left(M, T_{B^{\prime}}, M^{*}, T_{B}\right):$ In this case, a map in $\operatorname{Irr}_{\text {add } T}\left(T_{B}, M\right)$ is of degree 1. Assume the lifting is $f: \tau T_{B}[-1] \rightarrow M$. There is a non-split exact sequence $0 \rightarrow M \rightarrow$ $B^{\prime} \rightarrow M^{*} \rightarrow 0$. We have $\operatorname{dim} \operatorname{Hom}_{\mathcal{C}}\left(M^{*}, M[1]\right)=1$, by [BMRRT], and therefore $\operatorname{Hom}_{\mathscr{D}}\left(F M^{*}, M[1]\right)=\operatorname{Hom}_{\mathcal{D}}\left(\tau^{-1} M^{*}, M\right)=0$. Therefore, if $f: T_{B} \rightarrow M$ factors through $M^{*}[-1]$ in $\mathcal{C}$, there must be a map $g: \tau T_{B}[-1] \rightarrow M$ in $\mathscr{D}$, such that there is a commutative diagram

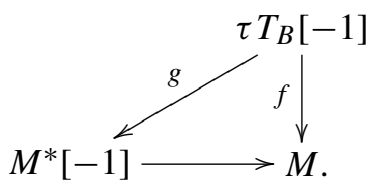

By Lemma 4.9, we have that $\operatorname{Hom}_{\mathscr{D}}\left(\tau T_{B}, M^{*}\right)=\operatorname{Hom}_{\mathscr{D}}\left(T_{B}, \tau^{-1} M^{*}\right)=0$, and thus we obtain $f=0$.

$\left(T_{B^{\prime}}, M^{*}, T_{B}, M\right)$ or $\left(M^{*}, T_{B}, M, T_{B^{\prime}}\right)$ : In these cases, a map in $\operatorname{Irr}_{\text {add } T}\left(T_{B}, M\right)$ is of degree 0 . Assume the lifting of it is $f: T_{B} \rightarrow M$. The preimage of $M^{*}$ in $\mathscr{D}$ is a module in these cases, so a factorisation of $f$ must be of the form

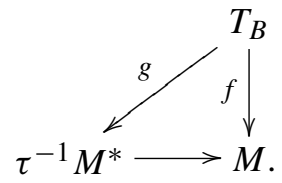

Lemma 4.9 gives $f=0$.

$\left(T_{B}, M, T_{B^{\prime}}, M^{*}\right):$ In this case the preimage of $M^{*}$ in $\mathscr{D}$ is either a module or $P$ [1], for an indecomposable projective $H$-module $P$. In both cases, a map in $\operatorname{Irr}_{\text {add } T}\left(T_{B}, M\right)$ is a map of degree 0 . Assume the lifting is $f: T_{B} \rightarrow M$. In the first case there is a non-split exact sequence $0 \rightarrow M \rightarrow B^{\prime} \rightarrow M^{*} \rightarrow 0$. Therefore, since $\operatorname{dim} \operatorname{Hom}_{\mathcal{C}}\left(M^{*}, M[1]\right)=1$, we have $\operatorname{Hom}_{\mathscr{D}}\left(\tau^{-1} M^{*}, M\right)=0$. Since $\operatorname{Hom}_{\mathcal{D}}\left(T_{B}, M^{*}[-1]\right)=0$, we must have $f=0$, if $f$ factors as below

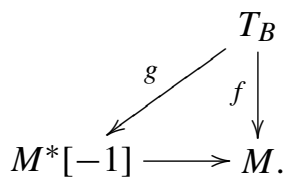

Assume $M^{*} \simeq P[1]$, with $P$ projective. If $\operatorname{Hom}_{\mathscr{D}}\left(T_{B}, P\right) \neq 0$, then $T_{B}$ is also projective. Therefore $\operatorname{Hom}_{\mathscr{D}}\left(T_{B}, P[1]\right)=0$, and thus $\operatorname{Hom}_{\mathcal{C}}\left(T_{B}, M^{*}\right)=0$, which means that $T_{B^{\prime}}$ is a zero-vertex in $\Gamma^{\prime}$, a contradiction. Then $\operatorname{Hom}_{\mathscr{D}}\left(T_{B}, P\right)=0$, but 
this means that $f: T_{B} \rightarrow M$ factors through $M^{*}[-1] \simeq P$ only for $f=0$. Thus, the map $\alpha$ is a monomorphism, and the sequence (4) is exact.

Thus, $\operatorname{dim} \widetilde{\operatorname{Hom}}\left(T_{B}, M^{*}\right)=\operatorname{dim} \widetilde{\operatorname{Hom}}\left(T_{B}, B^{\prime}\right)-\operatorname{dim} \widetilde{\operatorname{Hom}}\left(T_{B}, M\right)=t^{\prime} s-r$. This means that $\operatorname{dim}\left(\operatorname{Irr}_{\text {add } T^{\prime}}\left(T_{B}, T_{B^{\prime}}\right) \otimes_{K} \operatorname{Irr}_{\text {add } T^{\prime}}\left(T_{B^{\prime}}, M^{*}\right) \cap I\right)=r$, so $\Gamma^{\prime}$ is balanced at the vertex 3 , corresponding to $T_{B^{\prime}}$.

We now show that $\Gamma^{\prime}$ is balanced at the vertex 1 , corresponding to $T_{B}$, or 1 is a zero-vertex. Assume it is not a zero-vertex. We have the dual version of Lemma 4.9.

Lemma 4.10. Assume that $T_{B}$ does not correspond to a zero-vertex and that $M^{*}$ is before $T_{B}$ in the above ordering. Then $\operatorname{Hom}_{D}\left(M^{*}, \tau T_{B^{\prime}}\right)=0$.

Proof. Similar to the proof of Lemma 4.9.

Now, consider the triangle

$$
M^{*} \rightarrow B \rightarrow M \rightarrow
$$

We need to show that there is an exact sequence

$$
0 \rightarrow \widetilde{\operatorname{Hom}}\left(M, T_{B^{\prime}}\right) \rightarrow \widetilde{\operatorname{Hom}}\left(B, T_{B^{\prime}}\right) \rightarrow \widetilde{\operatorname{Hom}}\left(M^{*}, T_{B^{\prime}}\right) \rightarrow 0,
$$

where $\widetilde{\operatorname{Hom}}\left(M, T_{B^{\prime}}\right)=\operatorname{Irr}_{\text {add } T}\left(M, T_{B^{\prime}}\right)$, while $\widetilde{\operatorname{Hom}}\left(B, T_{B^{\prime}}\right)=\operatorname{Irr}_{\text {add } \bar{T}}\left(B, T_{B^{\prime}}\right)$ and $\widetilde{\operatorname{Hom}}\left(M^{*}, T_{B^{\prime}}\right)=\operatorname{Irr}_{\text {add } T^{\prime}}\left(M^{*}, T_{B^{\prime}}\right)$. The proof of this is parallel to the proof for the sequence (4), and therefore omitted. Using the exact sequence (5), one obtains that $\Gamma^{\prime}$ is balanced at the vertex 1 .

We summarise the results of this section.

Theorem 4.11. Let $\Gamma$ be a cluster-tilted algebra of rank 3 .

(a) $\Gamma$ is either hereditary, or it is cyclic.

(b) If $\Gamma$ is cyclic, then each vertex of $\Gamma$ is either balanced or a zero-vertex.

(c) Let $k$ be a vertex of $\Gamma$, let $\bar{\delta}_{k}(\Gamma)$ be the mutation in direction $k$, and let $k^{*}$ be the new vertex of $\bar{\delta}_{k}(\Gamma)$. Then there are the following possible cases:

I. Both $\Gamma$ and $\bar{\delta}_{k}(\Gamma)$ are hereditary.

II. $\Gamma$ is hereditary, while $\bar{\delta}_{k}(\Gamma)$ is cyclic with a zero-vertex at $k^{*}$,

III. $\Gamma$ is cyclic with a zero-vertex at $k$, and $\bar{\delta}_{k}(\Gamma)$ is hereditary, or

IV. $\Gamma$ is cyclic and balanced at $k$, and $\bar{\delta}_{k}(\Gamma)$ is cyclic and balanced at $k^{*}$.

Proof. This follows directly from the previous results in this section, and the fact that all cluster-tilted algebras can be obtained by starting with a hereditary algebra, and then performing a finite number of mutations [BMRRT], [BMR].

The above theorem is very easily verified for algebras of finite type, as indicated in the introduction of this section. 


\section{Mutation}

As mentioned in the introduction, in view of Proposition 3.2 it is possible to assign to a cluster-tilted algebra $\Gamma$ a skew-symmetric matrix $X_{\Gamma}=\left(x_{i j}\right)$. More precisely, if there is at least one arrow from $i$ to $j$ in the quiver of the endomorphism-algebra $\Gamma$, let $x_{i j}$ be the number of arrows from $i$ to $j$. If there are no arrows between $i$ and $j$, let $x_{i j}=0$. Let $x_{i j}=-x_{j i}$ otherwise.

Now let $\bar{T}$ be an almost complete tilting object in the cluster category $\mathcal{C}$ with complements $M$ and $M^{*}$. Let $T=\bar{T} \amalg M$, let $T^{\prime}=\bar{T} \amalg M^{*}$, let $\Gamma=\operatorname{End}_{\mathcal{C}}(T)^{\text {op }}$ and let $\Gamma^{\prime}=\operatorname{End}_{\mathcal{C}}\left(T^{\prime}\right)^{\text {op }}$. Then we want to show that the quivers of $\Gamma$ and $\Gamma^{\prime}$ are related by the cluster-mutation formula. We use the results of Section 4 to show this for cluster-tilted algebras of rank 3 , and Theorem 2.13 to extend to the general case. There is an independent proof of this for finite representation type in [CCS2].

Theorem 5.1. Let $H$ be a hereditary algebra, and let $\bar{T}, M, M^{*}, \Gamma$ and $\Gamma^{\prime}$ be as above. Then the quivers of $\Gamma$ and $\Gamma^{\prime}$, or equivalently the matrices $X_{\Gamma}$ and $X_{\Gamma^{\prime}}$, are related by the cluster mutation formula.

Proof. First, assume $H$ has rank 3. In case $H$ is not connected, the claim is easily checked. Assume $H$ is connected. Fix $k$, the vertex where we mutate. By Theorem 1.2, it is clear that $x_{i k}^{\prime}=-x_{i k}$ for $i=1,2,3$, and that $x_{k j}^{\prime}=-x_{k j}$ for $j=1,2,3$.

Now assume $i \neq k$ and $j \neq k$. By Theorem 4.11, there are four possible cases.

Case I: This happens if and only if $k$ is a source or a sink. In this case it is clear that either $x_{i k}=0$ or $x_{k j}=0$. For $i \neq k$ and $j \neq k$, it is clear that $x_{i j}=x_{i j}^{\prime}$, since in this case mutation at $k$ is the same as so-called APR-tilting at $k$. Thus the formula holds.

Case II: Since $k$ is now not a source or a sink, we can assume $\Gamma$ is the path algebra of

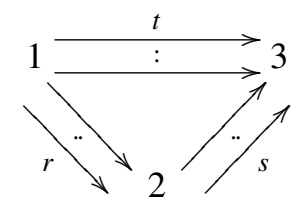

where $r>0$ and $s>0$ and $t \geq 0$ and with $k=2$. Then, by Lemma 4.7, the quiver of $\bar{\delta}_{2}(\Gamma)$ is

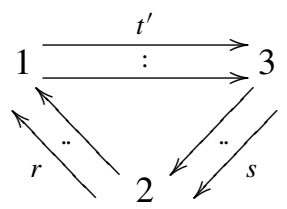


with $t^{\prime}=r s+t$. So $x_{13}^{\prime}=t^{\prime}=r s+t$, and

$$
x_{13}+\frac{\left|x_{12}\right| x_{23}+x_{12}\left|x_{23}\right|}{2}=t+\frac{r s+r s}{2}=t+r s .
$$

Case III: We assume that the quiver of $\Gamma$ is

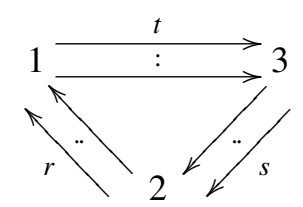

By Proposition 4.8, the quiver of $\Gamma^{\prime}$ is

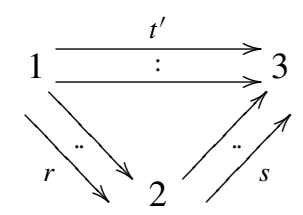

with $t^{\prime}=t-r s$. That is $x_{13}^{\prime}=t-r s$, and

$$
x_{13}+\frac{\left|x_{12}\right| x_{23}+x_{12}\left|x_{23}\right|}{2}=t+\frac{|-r|(-s)+(-r)|-s|}{2}=t-r s,
$$

and the formula holds.

Case IV: We assume the quiver of $\Gamma$ is the same as in case III. Now the quiver of $\Gamma^{\prime}$ is

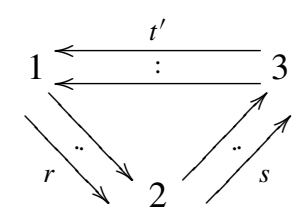

where $t^{\prime}=r s-t$. That is $x_{13}^{\prime}=-t^{\prime}=t-r s$, while

$$
x_{13}+\frac{\left|x_{12}\right| x_{23}+x_{12}\left|x_{23}\right|}{2}=t+\frac{|-r|(-s)+(-r)|-s|}{2}=t-r s,
$$

thus the formula holds true also in this case.

Now, assume that $H$ has arbitrary rank. Fix $k$, the vertex where we mutate. By Theorem 1.2, it is clear that $x_{i k}^{\prime}=-x_{i k}$ for any value of $i$, and that $x_{k j}^{\prime}=-x_{k j}$ for any value of $j$. Assume now that $k \neq i$ and $k \neq j$. Let $e_{i}, e_{j}, e_{k}$ be the primitive idempotents in $\Gamma$ corresponding to the vertices $i, j, k$ of the quiver of $\Gamma$. Assume $1_{\Gamma}=f+e_{i}+e_{j}+e_{k}$. and let $\Gamma_{\text {red }}=\Gamma / \Gamma f \Gamma$. Let $e_{i}, e_{j}, e_{k}^{*}$ be the primitive 
idempotents corresponding to the vertices $i, j, k^{*}$ of the quiver of $\Gamma^{\prime}$. Assume $1_{\Gamma^{\prime}}=$ $f^{\prime}+e_{i}+e_{j}+e_{k^{*}}$. and let $\Gamma_{\text {red }}^{\prime}=\Gamma^{\prime} / \Gamma^{\prime} f^{\prime} \Gamma^{\prime}$. It is clear that the number of arrows from $i$ to $j$ in the quiver of $\Gamma_{\text {red }}$ is $x_{i j}$ and the number of arrows from $i$ to $j$ in the quiver of $\Gamma_{\text {red }}^{\prime}$ is $x_{i j}^{\prime}$. So, by the first part of the proof, $x_{i j}$ and $x_{i j}^{\prime}$ are related by the matrix mutation formula.

There are some nice direct consequences of this. For the first one, one uses in addition to Theorem 5.1 that the tilting graph (see [BMRRT, Section 3]) is connected, [BMRRT, Prop. 3.5].

Corollary 5.2. Let $Q$ be a finite quiver with no oriented cycles. Then a quiver $Q^{\prime}$ can be obtained from $Q$ by repeated mutation if and only if $Q^{\prime}$ is the quiver of a cluster-tilted algebra $\operatorname{End}_{\mathcal{C}}(T)^{\mathrm{op}}$ for some tilting object $T$ in $\mathcal{C}=\mathcal{C}_{K Q}$.

We thank A. Seven for pointing out to us the following direct consequence of Theorem 2.13, and Corollary 5.2.

Corollary 5.3. Let $Q$ be a quiver which can be obtained by repeated mutation from an acyclic quiver. Then all full subquivers of $Q$ have the same property.

\section{Connections to cluster algebras}

Our main motivation for studying matrix mutation for quivers/matrices associated with tilting objects in cluster categories is the connection to cluster algebras. In this section we explain how Theorem 5.1 gives such a connection. In order to formulate our result we first need to give a short introduction to a special type of cluster algebras [FZ1], relevant to our setting [BFZ]. See also [FZ2] for an overview of the theory of cluster algebras.

Let $\mathcal{F}=\mathbb{Q}\left(u_{1}, \ldots, u_{n}\right)$ be the field of rational functions in indeterminates $u_{1}, \ldots, u_{n}$, let $\underline{x}=\left\{x_{1}, \ldots, x_{n}\right\} \subset F$ be a transcendence basis over $\mathbb{Q}$, and $B=\left(b_{i j}\right)$ an $n \times n$ skew-symmetric integer matrix. A pair $(\underline{x}, B)$ is called a seed. The cluster algebra associated to the seed $(\underline{x}, B)$ is by definition a certain subring $\mathcal{A}(\underline{x}, B)$ of $\mathcal{F}$, as we shall describe. Given such a seed $(\underline{x}, B)$ and some $i$, with $1 \leq i \leq n$, define a new element of $x_{i}^{\prime}$ of $\mathcal{F}$ by

$$
x_{i} x_{i}^{\prime}=\prod_{j ; b_{j i}>0} x^{b_{j i}}+\prod_{j ; b_{j i}<0} x^{-b_{j i}} .
$$

We say that $x_{i}, x_{i}^{\prime}$ form an exchange pair. We obtain a new transcendence basis $\underline{x^{\prime}}=\left\{x_{1}, \ldots, x_{n}\right\} \cup\left\{x_{i}^{\prime}\right\} \backslash\left\{x_{i}\right\}$ of $\mathcal{F}$. Then define a new matrix $B^{\prime}=\left(b_{i j}^{\prime}\right)$ associated 
with $B$ by

$$
b_{i j}^{\prime}= \begin{cases}-b_{i j} & \text { if } k=i \text { or } k=j, \\ b_{i j}+\frac{\left|b_{i k}\right| b_{k j}+b_{i k}\left|b_{k j}\right|}{2} & \text { otherwise. }\end{cases}
$$

The pair $\left(\underline{x^{\prime}}, B^{\prime}\right)$ is called the mutation of the seed $(\underline{x}, B)$ in direction $i$, written $\mu_{i}(\underline{x}, B)=\left(\underline{x}^{\prime}, B^{\prime}\right)$. Let $\&$ be the set of seeds obtained by iterated mutations of $(\underline{x}, B)$ (in all possible directions). The set of cluster variables is by definition the union of all transcendence bases appearing in all the seeds in $\delta$, and the cluster algebra $\mathcal{A}(\underline{x}, B)$ is the subring of $\mathcal{F}$ generated by the cluster variables. The transcendence bases appearing in the seeds are called clusters.

As mentioned earlier, there is a 1-1 correspondence between finite quivers with no loops and no oriented cycle of length two and skew-symmetric integer matrices (up to reordering the columns). The vertices of the quiver of a matrix $B=\left(b_{i j}\right)$ are $1, \ldots, n$, and there are $b_{i j}$ arrows from $i$ to $j$ if $b_{i j}>0$. The cluster algebra is said to be acyclic if there is some seed where the quiver associated with the matrix has no oriented cycles [BFZ]. We take the corresponding seed as an initial seed. In this case, let $H=K Q$ be the hereditary path algebra associated with an initial seed $(\underline{x}, B)$. Let $\mathcal{C}=\mathcal{C}_{H}$ be the corresponding cluster category, and let $T$ be a tilting object in $\mathcal{C}$. Similar to the above we can associate with $T$ a tilting seed $\left(T, Q_{T}\right)$, where $Q_{T}$ is the quiver of the endomorphism algebra $\operatorname{End}_{\mathcal{C}}(T)^{\text {op }}$. Let $T_{1}, \ldots, T_{n}$ be the non-isomorphic indecomposable direct summands of $T$. Fix $k$, and let as before $\delta_{k}(T)=T^{\prime}$ be the tilting object of $\mathcal{C}$ obtained by exchanging $T_{k}$ with $T_{k}^{*}$ (using our earlier notation from Theorem 1.2). Define mutation of $\left(T, Q_{T}\right)$ in direction $k$ to be given by $\delta_{k}\left(T, Q_{T}\right)=\left(T^{\prime}, Q_{T^{\prime}}\right)$.

We now want to associate tilting seeds with seeds for acyclic cluster algebras. We first associate $\left(H[1], Q_{H}\right)$ with a fixed initial seed $(\underline{x}, B)$, where $Q$ is the quiver for $B$ and $H=K Q$. Let $\left(\underline{x}^{\prime}, B^{\prime}\right)$ be some seed. We then have $\left(\underline{x}^{\prime}, B^{\prime}\right)=\mu_{k_{t}} \ldots \mu_{k_{1}}(\underline{x}, B)$ for some ordered sequence $\left(k_{1}, \ldots, k_{t}\right)$. There are in general several such sequences, and we choose one of minimal length. Associated with $(\underline{x}, B)$ is the sequence of length 0 , that is the empty set $\emptyset$. We define $\alpha((\underline{x}, B), \emptyset)=\left(H[1], Q_{H}\right)$, and $\alpha\left(\left(\underline{x}^{\prime}, B^{\prime}\right),\left(k_{1}, \ldots, k_{t}\right)\right)=\delta_{k_{t}} \ldots \delta_{k_{1}}\left(H[1], Q_{H}\right)=\left(T^{\prime}, Q_{T^{\prime}}\right)$. Fix an ordering on the cluster variables in the cluster $x=\left\{x_{1}, \ldots, x_{n}\right\}$ of the chosen initial seed and choose a corresponding indexing for the $H_{i}$ in $H=H_{1} \amalg \cdots \amalg H_{n}$, so that we have a correspondence between $x_{i}$ and $H_{i}$. This induces a correspondence between the cluster variables $x_{i}^{\prime}$ in the cluster $\underline{x}^{\prime}$ and the indecomposable direct summands $T_{i}^{\prime}$ in $T^{\prime}$, which we also denote by $\alpha$. We do not know in general if the definition of $\alpha$ only depends on the seed $\left(\underline{x}^{\prime}, B^{\prime}\right)$.

We can now formulate the connection between cluster algebras and tilting in cluster categories implied by our main result.

Theorem 6.1. Let the notation be as above, with $(\underline{x}, B)$ an initial seed for an acyclic cluster algebra, and $\left(T^{\prime}, Q_{T^{\prime}}\right)$ a tilting seed corresponding to a seed $\left(\underline{x}^{\prime}, B^{\prime}\right)$, via 
the correspondence $\alpha$, inducing a correspondence $x_{i}^{\prime} \leftrightarrow T_{i}^{\prime}$ for $x_{i}^{\prime} \in \underline{x}^{\prime}$ and $T_{i}^{\prime}$ an indecomposable direct summand of $T^{\prime}$.

(a) For any $k \in\{1, \ldots, n\}$ we have a commutative diagram

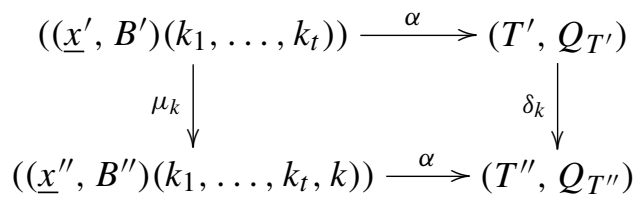

where $\underline{x}^{\prime \prime}$ is the cluster obtained from $\underline{x}^{\prime}$ by replacing $x_{k}^{\prime} \in \underline{x}^{\prime}$ by $x_{k}^{\prime \prime}$, and $T^{\prime \prime}$ is the tilting object in $\mathrm{C}$ obtained by exchanging the indecomposable summand $T_{k}^{\prime}$ by $T_{k}^{\prime \prime}$ where $T^{\prime}=\bar{T} \amalg T_{k}^{\prime}$ and $T^{\prime \prime}=\bar{T} \amalg T_{k}^{\prime \prime}$ are non-isomorphic tilting objects.

(b) Identifying $x_{k}^{\prime}$ with $T_{k}^{\prime}$ and $x_{k}^{\prime \prime}$ with $T_{k}^{\prime \prime}$, the multiplication rule for $x_{k}^{\prime} x_{k}^{\prime \prime}$ is given by

$$
T_{k}^{\prime} T_{k}^{\prime \prime}=\prod\left(T_{j}^{\prime}\right)^{a_{j}}+\prod\left(T_{l}^{\prime}\right)^{c_{l}}
$$

where $a_{j}$ and $c_{l}$ are determined by the minimal respectively right and left add $\bar{T}$ approximations $\amalg\left(T_{j}^{\prime}\right)^{a_{j}} \rightarrow T_{k}^{\prime}$ and $T_{k}^{\prime} \rightarrow \amalg\left(T_{l}^{\prime}\right)^{c_{l}}$.

Proof. (a): This follows by induction, using Theorem 5.1, where $\delta_{k}$ is interpreted as given by a mutation rule like $\mu_{k}$.

(b) Let $T_{k}^{\prime}$ be the direct summand of $T^{\prime}$ corresponding to $x_{k}^{\prime}$. By (a), $Q_{T^{\prime}}$ is the quiver of $B^{\prime}$, and the monomials $M_{1}$ and $M_{2}$ are given by the entries of the matrix $B^{\prime}$, hence by the arrows in the quiver $Q_{T^{\prime}}$. In particular, the arrows entering and leaving $k$, are given by the minimal right and minimal left add $\bar{T}$-approximations of $T_{k}^{\prime}$.

Note that with the appropriate formulation, this solves Conjecture 9.3 in [BMRRT].

For algebras of finite type we know from [BMRRT] that the map $\alpha$ gives a one-one correspondence between the seeds and tilting seeds, in particular it does not depend on the $t$-tuple $\left(i_{1}, \ldots, i_{t}\right)$. In fact, we have in this case a 1-1 correspondence between cluster variables and indecomposable objects of $\mathcal{C}$, inducing a 1-1 correspondence between clusters and tilting objects.

Two cluster variables $x_{k}$ and $x_{k}^{*}$ are said to form an exchange pair if there are $n-1$ cluster variables $\left\{y_{1}, \ldots, y_{n-1}\right\}$ such that $\left\{x_{k}, y_{1}, \ldots, y_{n-1}\right\}$ and $\left\{x_{k}^{*}, y_{1}, \ldots, y_{n-1}\right\}$ are clusters. Similarly we have exchange pairs with respect to tilting objects. If $\alpha$ identifies $x_{k}$ and $x_{k}^{*}$ with $T_{k}$ and $T_{k}^{*}$, respectively, we then have the following.

Theorem 6.2. For a cluster algebra of finite type, let $\alpha$ be the above correspondence between seeds and tilting seeds, and between cluster variables and indecomposable objects in the cluster category. 
(a) For any $k \in\{1, \ldots, n\}$ we have a commutative diagram

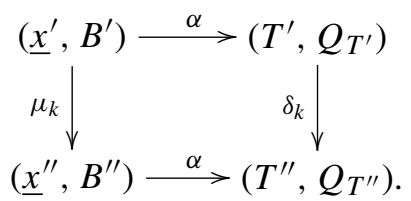

(b) Identify the cluster variables with the indecomposable objects in $\mathrm{C}$ via $\alpha$. We have

$$
T_{k} T_{k}^{*}=\prod\left(T_{j}\right)^{a_{j}}+\prod\left(T_{l}^{*}\right)^{c_{l}}
$$

for an exchange pair $T_{k}$ and $T_{k}^{*}$ where the $a_{j}$ and $c_{l}$ appear in the unique non-split triangles

$$
T_{k}^{*} \rightarrow \amalg T_{j}^{a_{j}} \rightarrow T_{k} \rightarrow
$$

and

$$
T_{i} \rightarrow \amalg T_{l}^{c_{l}} \rightarrow T_{i}^{*} \rightarrow
$$

in $\mathcal{C}$.

\section{References}

[APR] M. Auslander, M. I. Platzeck, I. Reiten, Coxeter functors without diagrams. Trans. Amer. Math. Soc. 250 (1979), 1-46. Zbl 0421.16016 MR 0530043

[AR] M. Auslander, I. Reiten, Representation theory of Artin algebras. III. Almost split sequences. Comm. Algebra 3 (1975), 239-294. Zbl 0331.16027 MR 0379599

[ARS] M. Auslander, I. Reiten, S. O. Smalø, Representation Theory of Artin Algebras. Cambridge Stud. Adv. Math. 36, Cambridge University Press, Cambridge 1995. Zbl 0834.16001 MR 1314422

[AS] M. Auslander, S. O. Smalø, Preprojective modules over Artin algebras. J. Algebra 66 (1980), 6-122. Zbl 0477.16013 MR 0591246

[BFZ] A. Berenstein, S. Fomin, A. Zelevinsky, Cluster Algebras III: Upper bounds and double Bruhat cells. Duke Math. J. 126 (2005), 1-52. Zbl 02147024 MR 2110627

[BES] D. Bessis, The dual braid monoid. Ann. Sci. Ecole Norm. Sup. (4) 36 (2003), 647-683. Zbl 1064.20039 MR 2032983

[B] K. Bongartz, Tilted algebras. In Representations of algebras (Puebla, 1980), Lecture Notes in Math. 903, Springer-Verlag, Berlin 1981, 26-38. Zbl 0478.16025 MR 0654701

[BMRRT] A. Buan, B. R. Marsh, M. Reineke, I. Reiten, G. Todorov, Tilting theory and cluster combinatorics. Adv. Math. 204 (2006), 572-618. Zbl 05047155 MR 2249625

[BMR] A. Buan, B. R. Marsh, I. Reiten, Cluster-tilted algebras. Trans. Amer. Math. Soc. 359 (2007), 323-332. Zbl 05120550 MR 2247893 
[BR] A. Buan, I. Reiten, Acyclic quivers of finite mutation type. Internat. Math. Res. Notices 13 (2006), article ID 12804, 1-10. Zbl 1116.16013 MR 2249997

[CC] P. Caldero, F. Chapoton, Cluster algebras as Hall algebras of quiver representations. Comment. Math. Helv. 81 (2006), 595-616. Zbl 1119.16013 MR 2250855

[CCS1] P. Caldero, F. Chapoton, R. Schiffler, Quivers with relations arising from clusters $\left(A_{n}\right.$ case). Trans. Amer. Math. Soc. 358 (2006), 1347-1364. Zbl 02237892 MR 2187656

[CCS2] P. Caldero, F. Chapoton, R. Schiffler, Quivers with relations and cluster-tilted algebras. Algebr. Represent. Theory 9 (2006), 359-376. Zbl 05125411 MR 2250652

[CK] P. Caldero, B. Keller, From triangulated categories to cluster algebras. Invent. Math., to appear. arXiv:math.RT/0506018

[CFZ] F. Chapoton, S. Fomin, A. Zelevinsky, Polytopal realizations of generalized associahedra, Canad. Math. Bull. 45 (2002), 537-566. Zbl 1018.52007 MR 1941227

[FG] V. V. Fock, A. B. Goncharov, Cluster ensembles, quantization and the dilogarithm. Preprint 2003/2007. arXiv:math.AG/0311245

[FZ1] S. Fomin, A. Zelevinsky, Cluster Algebras I: Foundations. J. Amer. Math. Soc. 15 (2002), 497-529. Zbl 1021.16017 MR 1887642

[FZ2] S. Fomin, A. Zelevinsky, Cluster Algebras: notes for the CDM-conference. In Current developments in mathematics, 2003, International Press, Somerville, MA, 2003. Zbl 1119.05108 MR 2132323

[FZ3] S. Fomin, A. Zelevinsky, $Y$-systems and generalized associahedra. Ann. of Math. (2) 158 (2003), 977-1018. Zbl 1057.52003 MR 2031858

[FZ4] S. Fomin, A. Zelevinsky, The Laurent phenomenon. Adv. in Appl. Math. 28 (2002), 119-144. Zbl 1012.05012 MR 1888840

[GSV1] M. Gekhtman, M. Shapiro, A. Vainshtein, Cluster algebras and Poisson geometry. Moscow Math. J. 3 (2003), 899-934. Zbl 1057.53064 MR 2078567

[GSV2] M. Gekhtman, M. Shapiro, A. Vainshtein, Cluster algebras and Weil-Petersson forms. Duke Math. J. 127 (2005), 291-311. Zbl 1079.53124 MR 2130414

[H] D. Happel, Triangulated categories in the representation theory of finite-dimensional algebras. London Math. Soc. Lecture Note Ser. 119, Cambridge University Press, Cambridge 1988. Zbl 0635.16017 MR 0935124

[H2] D. Happel, A characterization of hereditary categories with tilting object. Invent. Math. 144 (2001), 381-398. Zbl 1015.18006 MR 1827736

[HR] D. Happel, C. Ringel, Tilted algebras. Trans. Amer. Math. Soc. 274 (1982), 399-443. Zbl 0503.16024 MR 0675063

[HRS] D. Happel, J. Rickard, A. Schofield, Piecewise hereditary algebras. Bull. London Math. Soc. 20 (1988), 23-28. Zbl 0638.16018 MR 0916069

[HU] D. Happel, L. Unger, On the set of tilting objects in hereditary categories. In Representations of algebras and related topics, Fields Inst. Commun. 45, Amer. Math. Soc., Providence, RI, 2005, 141-159. Zbl 1082.16019 MR 2146246

[Ho] M. Hoshino, Modules without self-extensions and Nakayama's conjecture. Arch. Math. (Basel) 43 (1984), 493-500. Zbl 0547.16014 MR 0775735 
[K] B. Keller, On triangulated orbit categories. Doc. Math. 10 (2005), 551-581. Zbl 02238184 MR 2184464

[KR] B. Keller, I. Reiten, Cluster-tilted algebras are Gorenstein and stably Calabi-Yau. Adv. Math. 211 (2007), 123-151. Zbl 05144368 MR 2313531

[Ke] O. Kerner, Exceptional components of wild hereditary algebras. J. Algebra 152 (1997), 184-206. Zbl 0779.16006 MR 1190411

[Ke2] O. Kerner, Representations of wild quivers. In Representation theory of algebras and related topics (Mexico City, 1994), CMS Conf. Proc. 19, Amer. Math. Soc., Providence, RI, 1996, 65-107. Zbl 0863.16010 MR 1388560

[MRZ] B. Marsh, M. Reineke, A. Zelevinsky, Generalized associahedra via quiver representations. Trans. Amer. Math. Soc. 355 (2003), 4171-4186. Zbl 1042.52007 MR 1990581

[P] D. I. Panyushev, ad-nilpotent ideals of a Borel subalgebra: generators and duality. J. Algebra 274 (2004), 822-846. Zbl 1067.17005 MR 2043377

[RV] I. Reiten, M. van den Bergh, Noetherian hereditary categories satisfying Serre duality. J. Amer. Math. Soc. 15 (2002), 295-366. Zbl 0991.18009 MR 1887637

[Ric] J. L. Rickard, Derived categories and stable equivalence. J. Pure. Appl. Algebra 61 (1989), 303-317. Zbl 0685.16016 MR 1027750

[R] C. M. Ringel, Tame algebras and integral quadratic forms. Lecture Notes in Math. 1099, Springer-Verlag, Berlin 1984. Zbl 0546.16013 MR 0774589

[V] J. L. Verdier, Catégories dérivées, état 0. In Semin. Geom. Algebr. Bois-Marie, SGA 4 1/2, Lecture Notes in Math. 569, Springer-Verlag, Berlin 1977, 262-311. Zbl 0407.18008

[V2] J. L. Verdier, Des catégories dérivées des catégories abéliennes. Astérisque 239 (1996). Zbl 0882.18010 MR 1453167

[Z] A. Zelevinsky, Cluster algebras. Notes for 2004 IMCC (Chonju, Korea, August 2004), Preprint 2004. arXiv:math.RT/0407414

Received March 22, 2005; revised September 9, 2005

Aslak Bakke Buan, Institutt for matematiske fag, Norges teknisk-naturvitenskapelige universitet, 7491 Trondheim, Norway

E-mail: aslakb@math.ntnu.no

Bethany R. Marsh, Department of Pure Mathematics, University of Leeds, Leeds, LS2 9JT, UK

E-mail: marsh@maths.leeds.ac.uk

Idun Reiten, Institutt for matematiske fag, Norges teknisk-naturvitenskapelige universitet,

7491 Trondheim, Norway

E-mail: idunr@math.ntnu.no 